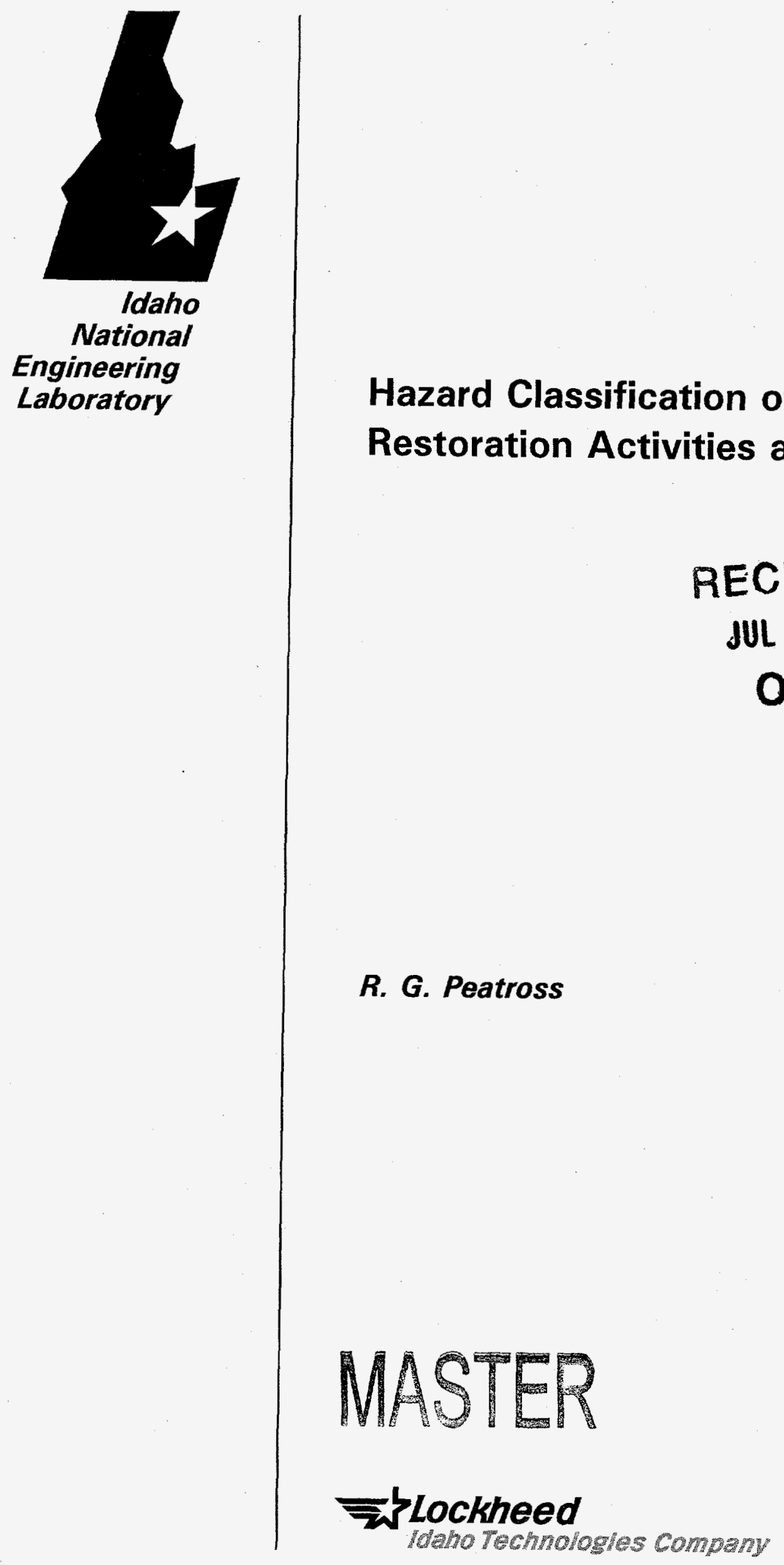

INEL-96/0054

Revision 0

April 1996

\title{
Hazard Classification of Environmental Restoration Activities at the INEL
}

\author{
RECEIVED \\ JUL $2^{9} 1996$ \\ OSTI
}

R. G. Peatross 
INEL-96/0054

Revision 0

\section{Hazard Classification for Environmental Restoration Activities at the INEL}

R. G. Peatross

Published April 1996

Idaho National Engineering Laboratory

Lockheed Martin Idaho Technology Idaho Falls, Idaho 83415

Prepared for the

U.S. Department of Energy

Under DOE Idaho Operations Office

Contract DE-AC07-94ID13223 
Hazard Classification of Environmental Restoration Activities at the INEL

INEL-96/0054

Revision 0

Prepared by:

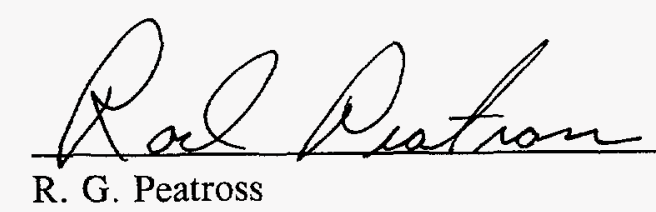

Chemical and Radiological Risk Assessments

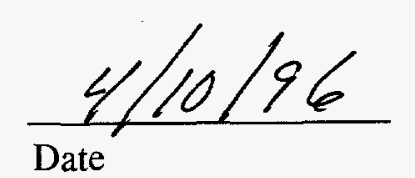

Date

Approved by:
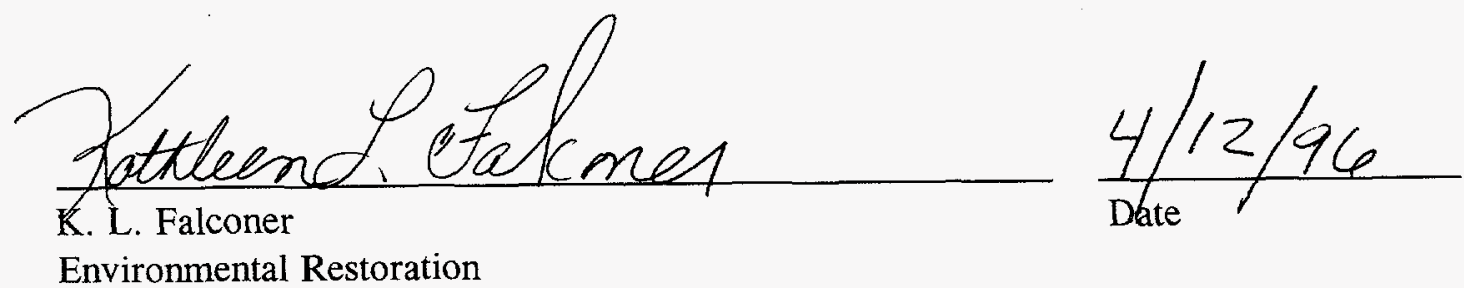


\section{DISCLAIMER}

Portions of this document may be illegible in electronic image products. Images are produced from the best available original document. 


\section{ABSTRACT}

This report presents the results of the hazard classification for environmental restoration of sites at the INEL. The hazard classification is based upon the approach recommended in Hazard Baseline Documentation (DOE-EM-STD-5502-94).

\section{DISCLAIMER}

This report was prepared as an account of work sponsored by an agency of the United States Government. Neither the United States Government nor any agency thereof, nor any of their employees, makes any warranty, express or implied, or assumes any legal liability or responsibility for the accuracy, completeness, or usefulness of any information, apparatus, product, or process disclosed, or represents that its use would not infringe privately owned rights. Reference herein to any specific commercial product, process, or service by trade name, trademark, manufacturer, or otherwise does not necessarily constitute or imply its endorsement, recommendation, or favoring by the United States Government or any agency thereof. The views and opinions of authors expressed herein do not necessarily state or reflect those of the United States Government or any agency thereof. 


\section{EXECUTIVE SUMMARY}

The following documents require that a hazard classification be prepared for all activities for which United States Department of Energy (DOE) has assumed environmental, safety, and health responsibility: The DOE Order 5481.1B, Safety Analysis and Review System and DOE Order 5480.23, Nuclear Safety Analysis Reports. A hazard classification defines the level of hazard posed by an operation or activity, assuming an unmitigated release of radioactive and nonradioactive hazardous material.

For environmental restoration activities, the release threshold criteria presented in Hazard Baseline Documentation (DOE-EM-STD-5502-94) are used to determine classifications, such as Radiological, Nonnuclear, and Other Industrial facilities.

Based upon DOE-EM-STD-5502-94, environmental restoration activities in all but one of the sites addressed by the scope of this classification (see Section 2) can be classified as "Other Industrial Facility." DOE-EM-STD-5502-94 states that a Health and Safety Plan and compliance with the applicable Occupational Safety and Health Administration (OSHA) standards are sufficient safety controls for this classification. 


\section{CONTENTS}

ABSTRACT $\ldots \ldots \ldots \ldots \ldots \ldots \ldots \ldots \ldots \ldots \ldots \ldots \ldots \ldots \ldots \ldots \ldots \ldots$

EXECUTIVE SUMMARY $\ldots \ldots \ldots \ldots \ldots \ldots \ldots \ldots \ldots \ldots \ldots$

1. INTRODUCTION $\ldots \ldots \ldots \ldots \ldots \ldots \ldots \ldots \ldots \ldots \ldots \ldots \ldots \ldots \ldots$

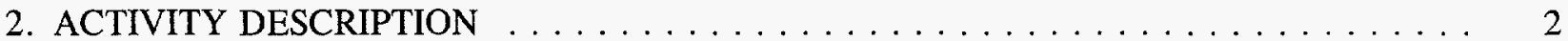

3. HAZARD CLASSIFICATION APPROACH $\ldots \ldots \ldots \ldots \ldots \ldots \ldots \ldots$

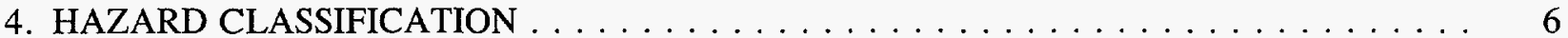

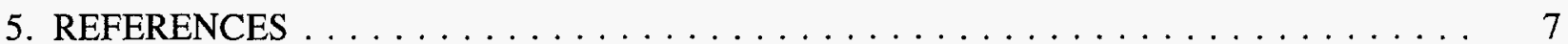

Appendix A-Contaminants and Sum-of-the-Ratio Calculations $\ldots \ldots \ldots \ldots \ldots \ldots$

FIGURE

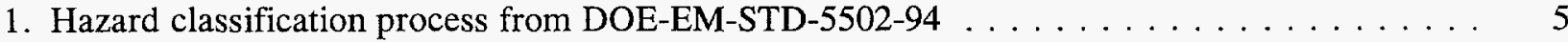

\section{TABLE}

1. Summary of WAGs encompassed by this classification $\ldots \ldots \ldots \ldots \ldots$ 



\section{Hazard Classification of Environmental Restoration Activities at the INEL}

\section{INTRODUCTION}

United States Department of Energy (DOE) Order 5481.1B, Safety Analysis and Review System ${ }^{1}$ and DOE Order 5480.23, Nuclear Safety Analysis Reports ${ }^{2}$ require that a hazard classification be prepared for all activities for which DOE has assumed environmental, safety, and health responsibility. A hazard classification defines the level of hazard posed only by potential releases of radiological and nonradiological hazardous materials from an operation or activity, assuming no mitigating systems are available.

DOE-EM-STD-5502-94 classifies facilities and activities as Nuclear, Nonnuclear, Radiological, and Other Industrial. ${ }^{3}$ The classifications are based only on the releasable quantity thresholds listed in 40 CFR $302,{ }^{4}$ DOE-STD-1027-92, 29 CFR $1910.119,{ }^{6}$ and 40 CFR $355 .{ }^{7}$ 


\section{ACTIVITY DESCRIPTION}

This classification is intended to envelop restoration activities performed by Environmental Restoration (ER) and their subcontractors. The majority of these activities take place outdoors and involve working with relatively small volumes of liquid, soil, and waste that may be contaminated with radionuclides, chemicals, or both.

ER activities are under the management of the ER directorate organization. The waste area groups (WAGs) and the areas that comprise each WAG are identified in Table 1. Some ER activities are excluded from using this hazard classification. Activities that are excluded include:

- $\quad$ Radioactive Waste Management Complex (RWMC), which has a Safety Analysis Report (SAR) that addresses activities inside the RWMC fence

- Areas where unexploded ordnance are present

- Any activity for which nuclear criticality has been identified as a valid concern.

Characterization activities include soil sampling; well drilling; liquid sampling from wells, ponds, or tanks; and sediment sampling from ponds, tanks, or the ground surface. These activities include a wide variety of accepted sampling methods and matrices and typically involve small volumes of material.

Remediation activities include soil removal using earthmoving equipment (backhoe, front-end loader, etc.) or by hand; cleanup of contaminated liquids; removal of waste (mixed) from underground storage tanks (USTs), in situ grouting of USTs, removal of USTs; and capping and monitoring of contaminated sites.

During ER activities, health and safety protection will be implemented by the Health and Safety Plan (HSP) written for each ER activity. The HSP provides specific guidelines to minimize health and safety risks to the worker and public. The HSPs are prepared in accordance with 29 CFR 1910.120, Hazardous Waste Operations and Emergency Response. 
Table 1. Summary of WAGs encompassed by this classification.

\begin{tabular}{|c|c|c|}
\hline WAG & Facility & Remarks \\
\hline 1 & Test Area North (TAN) & Includes: \\
\hline & & $\begin{array}{ll}\text { - } & \text { Technical Support Facility (TSF) } \\
\text { - } & \text { Initial Engine Test (IET) Facility } \\
\text { - } & \text { Containment Test Facility (CTF) } \\
\text { - } & \text { Specific Manufacturing Capability (SMC) Facility } \\
\text { - Water Reactor Research Test Facility (WRRTF). }\end{array}$ \\
\hline 2 & Test Reactor Area (TRA) & $\begin{array}{l}\text { Includes several deactivated reactors, the Advanced Test } \\
\text { Reactor and associated buildings, warm, cold, and sanitary } \\
\text { waste ponds located outside the fence line, and the } \\
\text { retention basin inside the fence line. }\end{array}$ \\
\hline 3 & $\begin{array}{l}\text { Idaho Chemical Processing Plant } \\
\text { (ICPP) }\end{array}$ & $\begin{array}{l}\text { Includes reprocessing facilities for defense and research } \\
\text { spent fuels. }\end{array}$ \\
\hline 4 & Central Facilities Area (CFA) & General support facility for the INEL. \\
\hline 5 & $\begin{array}{l}\text { Power Burst Facility (PBF)/ } \\
\text { Auxiliary Reactor Area (ARA) }\end{array}$ & $\begin{array}{l}\text { Includes several test reactors, some of which have been } \\
\text { partially or completely decontaminated and decommis- } \\
\text { sioned. }\end{array}$ \\
\hline 6 & $\begin{array}{l}\text { Experimental Breeder Reactor-I/ } \\
\text { Boiling Water Reactor } \\
\text { Experiment (EBR-I/BORAX) }\end{array}$ & Includes areas within the EBR-I and BORAX sites. \\
\hline 7 & RWMC & Includes activities outside the RWMC facility fence only. \\
\hline 10 & Miscellaneous sites & $\begin{array}{l}\text { Includes miscellaneous surface sites and liquid disposal } \\
\text { areas not included within other WAGs. }\end{array}$ \\
\hline $\mathrm{D} \& \mathrm{D}$ & D\&D Program facilities & Includes all aspects of D\&D \\
\hline
\end{tabular}




\section{HAZARD CLASSIFICATION APPROACH}

Figure 1 shows the DOE-EM-STD-5502-94 classification process recommended by DOE for all environmental management activities. This process was used to determine the appropriate hazard classification for ER activities.

The hazard classification process requires the following: (a) the contaminants and corresponding threshold quantities (TQs) in DOE-STD-1027, reportable quantities (RQs) in 40 CFR 302 Appendix B for radioisotopes and Table 40 CFR 302 for nonradioactive hazardous materials be identified, (b) an estimate of the quantities of contaminants at the remediation or characterization area, (c) a determination of the ratio of the assumed quantities to the TQs and RQs, (d) a sum of the ratios, (e) an adjustment of the sum of the ratios by a ratio of $1 \mathrm{E}-03$ (the most conservative release fraction used to determine the TQs and RQs) ${ }^{8}$ to a less conservative but defensible release fraction, and (f) use the actual release fractions found in Reference 8 for each radioisotope if $1.0 \mathrm{E}-03$ is too conservative for a site. Several conservative assumptions were used in arriving at the sum-of-the-ratios to ensure the enveloping of ER activities addressed by this hazard classification. These assumptions are discussed in the following paragraphs.

The data tables in Appendix A list the contaminants, the assumed concentrations in soil/waste and water, the associated TQs and RQs, and the adjusted sums of the ratios. The list of contaminants and the assumed concentrations are an aggregate of the maximum concentrations of recorded sample analysis results recorded for WAGs addressed by this hazard classification (see Section 2). ${ }^{9}$

It is assumed that the release volumes are $1 \mathrm{~m}^{3}$ of soil/waste and $4,000 \mathrm{~L}$ of water. The assumed volumes are high for characterization activities and most remediation activities and results in a much larger airborne release of material than would be expected. These assumed volumes will bound activities currently planned by ER for calender year 1996.

The release fraction for contaminated liquids is assumed to be the same as for contaminated soil. This assumption results in a much larger airborne release of material for a liquid than would be expected.

When summing the ratios, it is assumed that a single restoration activity will involve all the contaminants found to date and at the maximum concentrations measured to date. These conditions are assumed only to ensure that ER activities are enveloped by this hazard classification. The list of contaminants and the assumed concentrations should not be used as screening thresholds to determine if future ER activities are enveloped by this classification.

The hazard classification is segregated for operable units that because of high concentrations of contaminants result in radiological or nuclear facility hazard classifications rather than standard industrial. 


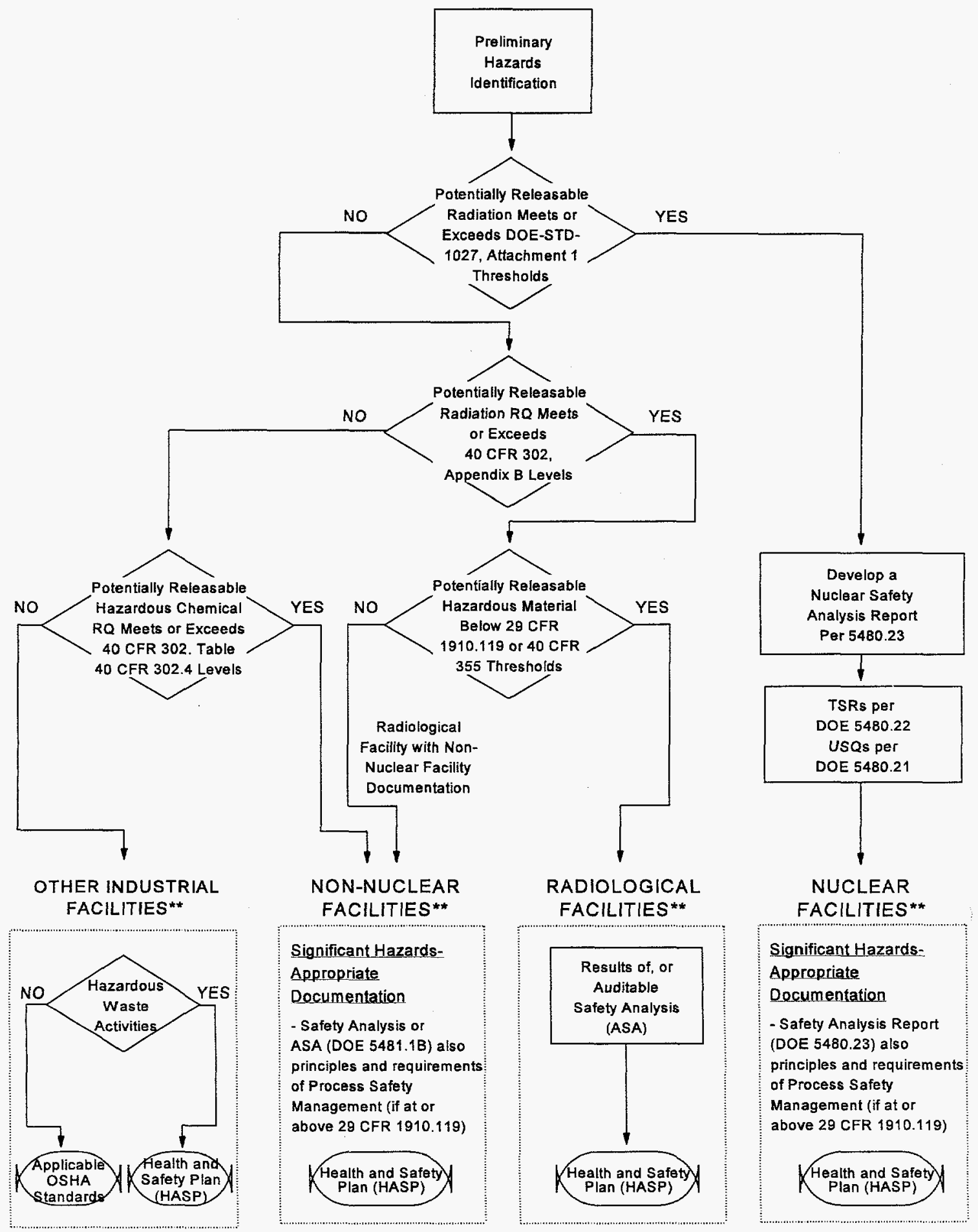

** Other types of safety and health hazard identification and control documentation may be necessary such as construction safety requirements, Chemical Hygiene Plans, and Hazcomm. All facilities with hazardous waste activities require a HASP as defined in 29 CFR 1910.120(a).

Figure 1. Hazard classification process from DOE-EM-STD-5502-94. 


\section{HAZARD CLASSIFICATION}

The sum of the ratios of releasable quantities compared to the TQs and RQs is less than 1.0 for all operable units (OUs) addressed by this hazard classification except OU 3-07 (see Appendix A). Therefore, ER activities in areas other than OU 3-07 and not excluded from this classification (see Section 2) may be classified as "Standard Industrial" and an auditable safety analysis or SAR is not needed.

Assuming a release volume of $1 \mathrm{~m}^{3}$, and the actual release fractions found in Reference 8, the sum of the ratios for OU 3-07 is less than one when compared to the TQs but is greater than one (actual value is two) when compared to the RQs. This site may still be classified as "Standard Industrial" only if the actual volume of potentially releasable soil is less than the volume assumed in the calculations; otherwise it is classified as "Radiological."

Excluding OU 3-07, the sum of the ratios will be less than one for the TQs and RQs even if the assumed volume of soil/waste or water contains all the radioactive and nonradioactive hazardous materials at the maximum (assumed) concentrations measured to date (1/96) for soil/waste and water. Adding the sums of the ratios for soil/waste and liquids equals 6E-04 when considering the TQs only (applies only to radionuclides) and 6E-01 when considering the RQs (radionuclides and hazardous materials). Therefore, the hazard classification will be "Standard Industrial."

This classification is conservative in the extreme and will envelope all typical characterization activities and most remediation activities at the INEL. The LMIT Chemical and Radiological Risk Assessment Department (CRRAD) should be consulted for all remediation activities to ensure that they are enveloped and for characterization activities involving large volumes of materials in a single container. Activity specific sum-of-the-ratio determinations may be required if there is a potential for a spill of volumes significantly greater than (i.e., a factor of two) $1 \mathrm{~m}^{3}$ for soils and wastes and 4,000 L for liquids. It must be noted that the volumes assumed in an activity-specific determination relate only to material spilled in a single event, not the total volume of material stored at a site, involved in a remedial action, or that may be spilled over the course of an action.

This hazard classification will be updated yearly by the CRRAD to include new sample data and to ensure that the assumed concentrations are still bounding. 


\section{REFERENCES}

1. U.S. Department of Energy, DOE Order 5481.1B, "Safety Analysis and Review System," September 23, 1986.

2. U.S. Department of Energy, DOE Order 5480.23, "Nuclear Safety Analysis Reports," April 30, 1992.

3. U.S. Department of Energy, "Hazard Baseline Documentation," DOE Limited Standard, DOE-EM-STD-5502-94, August 1994.

4. $\quad 40$ CFR 302.4, "Designation of Hazardous Substances."

5. DOE-STD-1027-92, "Hazard Classification and Accident Analysis Techniques for Compliance with DOE Order 5480.23, Nuclear Safety Analysis Reports," December 1992.

6. 29 CFR 1910.119, "Process Safety Management of Highly Hazardous Chemicals."

7. 40 CFR 355, "Emergency Planning and Notification."

8. ICF Incorporated, Technical Background Document to Support Final Rulemaking Pursuant to Section 102 of the Comprehensive Environmental Response, Compensation, and Liability Act: Radionuclides; A Report to the Emergency Response Division, Office of Emergency and Remedial Response, $U$. S. Environmental Protection Agency, February 1989.

9. LITCO (Lockheed Idaho Technologies Company), Auditable Safety Analysis for Environmental Restoration Activities at the INEL, INEL-95/0088, Revision 3, June 1995. 

Appendix A

Contaminants and Sum-of-the-Ratio Calculations 
A-2 


\section{Appendix A}

\section{Contaminants and Sum-of-the-Ratio Calculations}

\section{INTRODUCTION}

This appendix provides the basis for determining the hazard classification of ER activities. Tables A-1 through A-5 list the contaminants, the maximum measured concentrations of each contaminant, the nuclear facility threshold quantities (TQs) from DOE-STD-1027-92, the reportable quantities (RQs) from 40 CFR 302 Appendix B for radionuclides, and Table 40 CFR 302 for nonradioactive hazardous materials, the ratio of the RQs to the maximum quantities, and the sum of the ratios.

The list of contaminants and their maximum concentrations are from about six years of sample and analysis data reported in decision document packages, Track 2 preliminary scoping packages, hazard classifications, historical data reports, record of decisions, feasibility study reports, remedial investigation/feasibility study reports, treatability studies, Health and Safety Plans, Track 2 summary reports, and closure reports for operable units within the waste area groups addressed by this hazard classification.

Several equations were used to derive data in Tables A-1 through A-5. Some required a soil density value or a factor to adjust the TQs and RQs assuming a less conservative but defensible release fraction. The assumed soil density was taken from Reference A-1 and the assumed release fraction is from a draft EM hazard categorization standard that is based on Reference A-2. Although still draft at the time of this report, the EM standard has been through final review and comment resolution and is waiting issuance (per tele-con with Howard Goldin, author of the standard).

The assumed soil density for all sites at the INEL is $1.5 \mathrm{~g} / \mathrm{cm}^{3}\left(1,500 \mathrm{~kg} / \mathrm{m}^{3}\right)$. An airborne release fraction (ARF) of 5.0E-06 is assumed for a spill of soils/wastes and liquids. For all but one site, the sum of the ratios is adjusted by the ratio of this ARF to an ARF of $1.0 \mathrm{E}-03$ which is the most conservative value used to calculate the TQs and the RQs. ${ }^{\mathrm{A}-3}$ Because of relatively high soil concentrations, assuming a release fraction of 1E-03 for all radionuclides at the OU 3-7 site results in an overly conservative classification. For this site only, the actual release fractions found in Reference A-3 for each radionuclide were used in determining the hazard classification (see Table A-5). A volume of $1 \mathrm{~m}^{3}$ is assumed for soil/waste and a volume of $4,000 \mathrm{~L}$ is assumed for liquid. The assumed volumes are based on the approximate volume of a front-end loader bucket and the representative volume of water purged and collected during ground water sampling. Equation (1) through Equation (4) were used to calculate the data listed in Table A-1 through Table A-5. 
Table A-1. Values for radioactive material in soil or waste.

\begin{tabular}{|c|c|c|c|c|c|c|}
\hline Contaminant & $\begin{array}{l}\text { Assumed soil } \\
\text { concentration } \\
\text { (pCi/g) }\end{array}$ & $\begin{array}{c}\text { Assumed } \\
\text { quantity } \\
\text { (Ci) }\end{array}$ & $\begin{array}{c}\text { Category } 3 \text { TQs } \\
(\mathrm{Ci}) \\
\end{array}$ & $\begin{array}{l}\text { Ratio of assumed } \\
\text { quantity to the IQ }\end{array}$ & $\begin{array}{l}\text { RQs } \\
(\mathrm{Ci}) \\
\end{array}$ & $\begin{array}{l}\text { Ratio of assumed } \\
\text { quantity to the } \\
\mathrm{RQ}\end{array}$ \\
\hline Actinium-227 & $9.50 \mathrm{E}-03$ & $1.43 \mathrm{E}-08$ & $8.00 \mathrm{E}-01$ & $178 \mathrm{E}-08$ & $1.00 \mathrm{E}-03$ & $1,43 \mathrm{E}-05$ \\
\hline Actinium-228 & $1.50 \mathrm{E}+00$ & $2.25 \mathrm{E}-06$ & $1.00 \mathrm{E}+01$ & $225 \mathrm{E}-07$ & $1.00 \mathrm{E}+01$ & $225 \mathrm{E}-07$ \\
\hline Americium-241 & $3.20 \mathrm{E}+03$ & $4.80 \mathrm{E}-03$ & $1.00 \mathrm{E}+01$ & $480 \mathrm{E}-04$ & $1.00 \mathrm{E}-02$ & $480 \mathrm{E}-0 \mathrm{~T}$ \\
\hline Americium-242m & $2.40 \mathrm{E}-04$ & $3.60 \mathrm{E}-10$ & $1.00 \mathrm{E}+01$ & $3.60 \mathrm{E} \mathrm{I}$ & $1.00 E-02$ & $3,60 \mathrm{E}-08$ \\
\hline Americium-243 & $4.10 \mathrm{E}-01$ & $6.15 \mathrm{E}-07$ & $1.00 \mathrm{E}+01$ & $615 E 08$ & $1.00 \mathrm{E}-02$ & $615 \mathrm{E}-0 \mathrm{~S}$ \\
\hline Antimony-124 & ND & NA & $3.60 \mathrm{E}+02$ & $\mathrm{NA}$ & $1.00 \mathrm{E}+01$ & $\mathrm{NA}$ \\
\hline Antimony-125 & $2.40 \mathrm{E}+03$ & $3.60 \mathrm{E}-03$ & $1.20 \mathrm{E}+02$ & $300 \mathrm{E}-05$ & $1.00 \mathrm{E}+01$ & $3.60 \mathrm{E}-04$ \\
\hline Antimony-126 & $2.50 \mathrm{E}-02$ & $3.75 \mathrm{E}-08$ & $2.80 \mathrm{E}+02$ & $134 \mathrm{E} 10$ & $1.00 \mathrm{E}+01$ & $375 \mathrm{E}-09$ \\
\hline Bismuth-214 & $9.00 \mathrm{E}-01$ & 1.35E-06 & $8.20 \mathrm{E}+02$ & $165 \mathrm{E}-09$ & $1.00 \mathrm{E}+02$ & $135 \mathrm{E} 08$ \\
\hline Californium-252 & $6.90 \mathrm{E}+02$ & $1.04 \mathrm{E}-03$ & $3.20 \mathrm{E}+00$ & $323 \mathrm{E}=04$ & $1.00 \mathrm{E}-01$ & $104 \mathrm{E}-02$ \\
\hline Cerium-141 & ND & NA & $1.00 \mathrm{E}+03$ & $\mathrm{NA}$ & $1.00 \mathrm{E}+01$ & $\mathrm{NA}$ \\
\hline Cerium-144 & $1.40 \mathrm{E}+03$ & $2.10 \mathrm{E}-03$ & $1.00 \mathrm{E}+02$ & $210 \mathrm{E} \cdot 05$ & $1.00 \mathrm{E}+00$ & $2,10 \mathrm{E}-03$ \\
\hline Cesium-134 & $2.40 \mathrm{E}+04$ & $3.60 \mathrm{E}-02$ & $1.54 \mathrm{E}+03$ & $234 \mathrm{E}-05$ & $1.00 \mathrm{E}+00$ & $3.60 \mathrm{E}-02$ \\
\hline Cesium-135 & 4.30E-01 & $6.45 \mathrm{E}-07$ & $1.58 \mathrm{E}+03$ & $4.08 \mathrm{E}-10$ & $1.00 \mathrm{E}+01$ & $6.45 \mathrm{E}-08$ \\
\hline Cesium-137 & $4.80 \mathrm{E}+04$ & $7.20 \mathrm{E}-02$ & $1.38 \mathrm{E}+05$ & $522 \mathrm{E}-07$ & $1.00 \mathrm{E}+00$ & $720 \mathrm{E}-02$ \\
\hline Chromium-51 & $7.30 \mathrm{E}+00$ & $1.10 \mathrm{E}-05$ & $2.20 \mathrm{E}+04$ & $498 \mathrm{E}-10$ & $1.00 \mathrm{E}+03$ & $110 \mathrm{E}-08$ \\
\hline Cobalt -57 & $1.00 \mathrm{E}+00$ & $1.50 \mathrm{E}-06$ & $6.00 \mathrm{E}+03$ & $250 \mathrm{E}-10$ & $1.00 \mathrm{E}+02$ & $150 \mathrm{E}-08$ \\
\hline Cobalt-58 & ND & NA & $9.00 \mathrm{E}+02$ & $\mathrm{NA}$ & $1.00 \mathrm{E}+01$ & $\mathrm{NA}$ \\
\hline Cobalt- 60 & $3.40 \mathrm{E}+03$ & $5.10 \mathrm{E}-03$ & $2.80 \mathrm{E}+02$ & $182 \mathrm{E}-05$ & $1.00 \mathrm{E}+01$ & $510 \mathrm{E}-04$ \\
\hline Curium-242 & $2.20 \mathrm{E}-01$ & $3.30 \mathrm{E}-07$ & $3.20 \mathrm{E}+01$ & $103 \mathrm{E}-08$ & $1.00 \mathrm{E}+00$ & $330 \mathrm{E}-07$ \\
\hline Curium-243 & $3.30 \mathrm{E}-06$ & $4.95 \mathrm{E}-12$ & $8.20 \mathrm{E}+00$ & $6.04 \mathrm{E}-13$ & $1.00 \mathrm{E}-02$ & $495 \mathrm{E}-10$ \\
\hline Curium-244 & $8.00 \mathrm{E}+00$ & $1.20 \mathrm{E}-05$ & $1.05 \mathrm{E}+00$ & $114 \mathrm{E}-05$ & $1.00 \mathrm{E}-02$ & $120 \mathrm{E}-03$ \\
\hline Europium-152 & $8.80 \mathrm{E}+04$ & $1.32 \mathrm{E}-01$ & $2.00 \mathrm{E}+02$ & $6.60 \mathrm{E}-04$ & $1.00 \mathrm{E}+01$ & $1.32 \mathrm{E}-02$ \\
\hline Europium-154 & $6.40 \mathrm{E}+02$ & $9.60 \mathrm{E}-04$ & $2.00 \mathrm{E}+02$ & $480 \mathrm{E}-06$ & $1.00 \mathrm{E}+01$ & $9,60 \mathrm{E} \sim 05$ \\
\hline Europium-155 & $9.60 \mathrm{E}+03$ & $1.44 \mathrm{E}-02$ & $2.00 \mathrm{E}+02$ & $720 \mathrm{E}-0 \mathrm{~s}$ & $1.00 \mathrm{E}+01$ & $1.44 \mathrm{E}-03$ \\
\hline Francium-223 & $1.80 \mathrm{E}-06$ & $2.70 \mathrm{E}-12$ & $8.20 \mathrm{E}+03$ & $3,29 \mathrm{E} 16$ & $1.00 \mathrm{E}+02$ & $2,70 \mathrm{E}-14$ \\
\hline Hafnium-181 & ND & NA & $7.60 \mathrm{E}+02$ & $\mathrm{NA}$ & $1.00 \mathrm{E}+01$ & $\mathrm{NA}$ \\
\hline Iodine-129 & $1.60 \mathrm{E}+00$ & $2.40 \mathrm{E}-06$ & $6.00 \mathrm{E}-02$ & $400 \mathrm{E} 05$ & $1.00 \mathrm{E}-03$ & $240 \mathrm{E}-03$ \\
\hline Iron-59 & $5.30 \mathrm{E}-02$ & $7.95 \mathrm{E}-08$ & $6.00 \mathrm{E}+02$ & $133 \mathrm{E}-10$ & $1.00 \mathrm{E}+01$ & $7.95 \mathrm{E}-09$ \\
\hline Krypton-85 & $6.90 \mathrm{E}+02$ & $1.04 \mathrm{E}-03$ & $2.00 \mathrm{E}+04$ & $5.18 \mathrm{E}-08$ & $1.00 \mathrm{E}+03$ & $104 \mathrm{E}-06$ \\
\hline Lead-210 & $9.00 \mathrm{E}-04$ & $1.35 \mathrm{E}-09$ & $3.60 \mathrm{E}-01$ & $3.75 \mathrm{E}-09$ & $1.00 \mathrm{E}-02$ & $135 \mathrm{E}-07$ \\
\hline Lead- 212 & $1.00 \mathrm{E}+00$ & $1.50 \mathrm{E}-06$ & $3.20 \mathrm{E}+02$ & $4.69 \mathrm{E}-0.9$ & $1.00 \mathrm{E}+01$ & $150 \mathrm{E}-07$ \\
\hline Lead-214 & $1.40 \mathrm{E}+00$ & $2.10 \mathrm{E}-06$ & $8.20 \mathrm{E}+03$ & $256 \mathrm{E}-10$ & $1.00 \mathrm{E}+02$ & $210 \mathrm{E}-08$ \\
\hline Manganese-54 & $1.60 \mathrm{E}-01$ & $2.40 \mathrm{E}-07$ & $8.80 \mathrm{E}+02$ & $273 \mathrm{E}-10$ & $1.00 \mathrm{E}+01$ & $240 \mathrm{E}-08$ \\
\hline Mercury-203 & ND & NA & $3.60 \mathrm{E}+02$ & $\mathrm{NA}$ & $1.00 \mathrm{E}+01$ & $\mathrm{NA}$ \\
\hline Neptunium-237 & $5.50 \mathrm{E}+00$ & $8.25 \mathrm{E}-06$ & $4.20 \mathrm{E}-01$ & $196 \mathrm{E}-05$ & $1.00 \mathrm{E}-02$ & $8,25 \mathrm{E}-04$ \\
\hline Niobium-93m & $8.10 \mathrm{E}-01$ & $1.22 \mathrm{E}-06$ & $2.00 \mathrm{E}+03$ & $6.08 \mathrm{E}-10$ & $1.00 \mathrm{E}+02$ & $1222 \mathrm{E} 08$ \\
\hline Niobium-94 & $5.90 \mathrm{E}+00$ & $8.85 \mathrm{E}-06$ & $2.00 \mathrm{E}+02$ & $4,43 \mathrm{E}-08$ & $1.00 \mathrm{E}+01$ & $885 \mathrm{E}-07$ \\
\hline Niobium-95 & $3.80 \mathrm{E}+00$ & $5.70 \mathrm{E}-06$ & $9.60 \mathrm{E}+02$ & $594 \mathrm{E}-09$ & $1.00 \mathrm{E}+01$ & $570 \mathrm{E}-07$ \\
\hline Niobium-97 & ND & NA & $1.48 \mathrm{E}+03$ & $\mathrm{NA}$ & $1.00 \mathrm{E}+01$ & $\mathrm{NA}$ \\
\hline Palladium-107 & $7.40 \mathrm{E}-03$ & $1.11 \mathrm{E}-08$ & $4.20 \mathrm{E}+03$ & $2.64 \mathrm{E}-12$ & $1.00 \mathrm{E}+02$ & $111 \mathrm{E}-10$ \\
\hline Plutonium-238 & $1.90 \mathrm{E}+02$ & $2.85 \mathrm{E}-04$ & $6.20 \mathrm{E}-01$ & $4.60 \mathrm{E}=04$ & $1.00 \mathrm{E}-02$ & $2.85 \mathrm{E}-02$ \\
\hline Plutonium-239/240 & $4.60 \mathrm{E}+02$ & $6.90 \mathrm{E}-04$ & $5.20 \mathrm{E}-01$ & $1.33 \mathrm{E}=03$ & $1.00 \mathrm{E}-02$ & $6.90 \mathrm{E}-02$ \\
\hline Plutonium-241 & $2.00 \mathrm{E}+01$ & $3.00 \mathrm{E}-05$ & $3.20 \mathrm{E}+01$ & $938 \mathrm{E}-07$ & $1.00 \mathrm{E}+00$ & $300 \mathrm{E} 05$ \\
\hline Potassium-40 & $3.20 \mathrm{E}+01$ & $4.80 \mathrm{E}-05$ & $1.70 E+02$ & $2.82 \mathrm{E}-07$ & $1.00 \mathrm{E}+00$ & $4.80 \mathrm{E}-05$ \\
\hline
\end{tabular}


Table A-1. (continued).

\begin{tabular}{|c|c|c|c|c|c|c|}
\hline Contaminant & $\begin{array}{l}\text { Assumed soil } \\
\text { concentration } \\
(\mathrm{pCi} / \mathrm{g})\end{array}$ & $\begin{array}{l}\text { Assumed } \\
\text { quantity } \\
\text { (Ci) }\end{array}$ & $\begin{array}{c}\text { Category } 3 \text { TQs } \\
\text { (Ci) }\end{array}$ & $\begin{array}{l}\text { Ratio of assumed } \\
\text { quantity to the } 10\end{array}$ & $\begin{array}{l}\text { RQs } \\
(\mathrm{Ci})\end{array}$ & $\begin{array}{l}\text { Ratio of assumed } \\
\text { quantity to the } \\
\text { RQ }\end{array}$ \\
\hline Promethium-147 & $2.00 \mathrm{E}+01$ & $3.00 \mathrm{E}-05$ & $1.00 \mathrm{E}+03$ & $300 \mathrm{E}-08$ & $1.00 \mathrm{E}+01$ & $300 \mathrm{E} 06$ \\
\hline Protactinium-231 & $2.20 \mathrm{E}-02$ & $3.30 \mathrm{E}-08$ & $2.00 \mathrm{E}-01$ & $1.65 \mathrm{E}-07$ & $1.00 \mathrm{E}-02$ & $330 \mathrm{E}-06$ \\
\hline Protactinium-234 & $2.50 \mathrm{E}-04$ & $3.75 \mathrm{E}-10$ & $1.52 \mathrm{E}+03$ & $247 \mathrm{E} 13$ & $1.00 \mathrm{E}+01$ & $375 \mathrm{E}-11$ \\
\hline Radium-226 & $6.30 \mathrm{E}+00$ & $9.45 \mathrm{E}-06$ & $1.20 \mathrm{E}+01$ & $788 \mathrm{E}-07$ & $1.00 \mathrm{E}-01$ & $9.45 \mathrm{E}-05$ \\
\hline Radon-222 & ND & NA & $1.00 \mathrm{E}+01$ & $\mathrm{NA}$ & $1.00 \mathrm{E}-01$ & $\mathrm{NA}$ \\
\hline Rhodium-106/106m & ND & NA & $1.96 \mathrm{E}+03$ & $\mathrm{NA}$ & $1.00 \mathrm{E}+01$ & $\mathrm{NA}$ \\
\hline Ruthenium-103 & ND & NA & $1.56 \mathrm{E}+03$ & $\mathrm{NA}$ & $1.00 \mathrm{E}+01$ & $\mathrm{NA}$ \\
\hline Ruthenium-106 & $5.40 \mathrm{E}+01$ & $8.10 \mathrm{E}-05$ & $1.04 \mathrm{E}+02$ & $779 \mathrm{E}-07$ & $1.00 \mathrm{E}+00$ & $810 \mathrm{E}-05$ \\
\hline Samarium-151 & $2.50 \mathrm{E}+02$ & $3.75 \mathrm{E}-04$ & $1.00 \mathrm{E}+03$ & $3,75 \mathrm{E} 07$ & $1.00 \mathrm{E}+01$ & $375 \mathrm{E}-05$ \\
\hline Scandium-46 & $3.60 \mathrm{E}-01$ & $5.40 \mathrm{E}-07$ & $3.60 \mathrm{E}+02$ & $150 \mathrm{E} 09$ & $1.00 \mathrm{E}+01$ & $5,40 \mathrm{E}-08$ \\
\hline Silver-108m & $6.70 \mathrm{E}+01$ & $1.01 \mathrm{E}-04$ & $2.00 \mathrm{E}+02$ & $503 \mathrm{E}-07$ & $1.00 \mathrm{E}+01$ & $101 \mathrm{E}-05$ \\
\hline Silver-110m & ND & $\mathrm{NA}$ & $2.60 \mathrm{E}+02$ & $\mathrm{NA}$ & $1.00 \mathrm{E}+01$ & $\mathrm{NA}$ \\
\hline Strontium-90 & $3.80 \mathrm{E}+04$ & $5.70 \mathrm{E}-02$ & $1.60 \mathrm{E}+01$ & $3.56 \mathrm{E}-03$ & $1.00 \mathrm{E}-01$ & 570E-01 \\
\hline Tantalum-182 & ND & $\mathrm{NA}$ & $6.20 \mathrm{E}+02$ & $\mathrm{NA}$ & $1.00 \mathrm{E}+01$ & $\mathrm{NA}$ \\
\hline Technetium-99 & $6.90 \mathrm{E}+00$ & $1.04 \mathrm{E}-05$ & $1.78 \mathrm{E}+03$ & $581 E-09$ & $1.00 \mathrm{E}+01$ & $104 \mathrm{E}-06$ \\
\hline Tellurium-125m & $1.80 \mathrm{E}-01$ & $2.70 \mathrm{E}-07$ & $7.20 \mathrm{E}+02$ & $375 \mathrm{E} 10$ & $1.00 \mathrm{E}+01$ & $270 \mathrm{E}-08$ \\
\hline Thorium-228 & $3.40 \mathrm{E}+00$ & $5.10 \mathrm{E}-06$ & $1.00 \mathrm{E}+00$ & $510 \mathrm{E}-06$ & $1.00 \mathrm{E}-02$ & $510 \mathrm{E}-04$ \\
\hline Thorium-230 & $2.60 \mathrm{E}+00$ & $3.90 \mathrm{E}-06$ & $6.20 \mathrm{E}-01$ & $629 \mathrm{E}-06$ & $1.00 \mathrm{E}-02$ & $390 \mathrm{E}-04$ \\
\hline Thorium-232 & $2.80 \mathrm{E}+00$ & $4.20 \mathrm{E}-06$ & $1.00 \mathrm{E}-01$ & $420 \mathrm{E}-05$ & $1.00 \mathrm{E}-03$ & $420 \mathrm{E}-03$ \\
\hline Thorium-234 & $1.50 \mathrm{E}+02$ & $2.25 \mathrm{E}-04$ & $2.80 \mathrm{E}+03$ & $8.04 \mathrm{E}, 08$ & $1.00 \mathrm{E}+02$ & $225 \mathrm{E}-06$ \\
\hline Tin- $121 \mathrm{~m}$ & $2.70 \mathrm{E}-02$ & $4.05 \mathrm{E}-08$ & $1.78 \mathrm{E}+03$ & $2.28 \mathrm{E}-11$ & $1.00 \mathrm{E}+01$ & $4.05 \mathrm{E}-09$ \\
\hline Tin- 126 & $1.80 \mathrm{E}-01$ & $2.70 \mathrm{E}-07$ & $1.70 \mathrm{E}+02$ & $159 \mathrm{E} 09$ & $1.00 \mathrm{E}+00$ & $270 \mathrm{E}-07$ \\
\hline Tritium-3 & $4.50 \mathrm{E}+03$ & $6.75 \mathrm{E}-03$ & $1.00 \mathrm{E}+03$ & $675 \mathrm{E}-06$ & $1.00 \mathrm{E}+02$ & $675 \mathrm{E}-05$ \\
\hline Uranium-232 & $1.50 \mathrm{E}+02$ & $2.25 \mathrm{E}-04$ & $8.20 \mathrm{E}-01$ & $2,74 \mathrm{E}-04$ & $1.00 \mathrm{E}-02$ & $225 \mathrm{E}-02$ \\
\hline Uranium-233 & $1.20 \mathrm{E}+00$ & $1.80 \mathrm{E}-06$ & $4.20 \mathrm{E}+00$ & $429 \mathrm{E}-07$ & $1.00 \mathrm{E}-01$ & $180 \mathrm{E}-05$ \\
\hline Uranium-234 & $1.10 \mathrm{E}+03$ & $1.65 \mathrm{E}-03$ & $4.20 \mathrm{E}+00$ & $393 E=04$ & $1.00 \mathrm{E}-01$ & $165 \mathrm{~F}-02$ \\
\hline Uranium-235 & $1.40 \mathrm{E}+02$ & $2.10 \mathrm{E}-04$ & $4.20 \mathrm{E}+00$ & $500 \mathrm{E}-05$ & $1.00 \mathrm{E}-01$ & $210 \mathrm{E}-03$ \\
\hline Uranium-236 & $7.60 \mathrm{E}-01$ & $1.14 \mathrm{E}-06$ & $4.20 \mathrm{E}+00$ & $271 \mathrm{E}-67$ & $1.00 \mathrm{E}-01$ & $114 \mathrm{E}-05$ \\
\hline Uranium-238 & $5.20 \mathrm{E}+01$ & $7.80 \mathrm{E}-05$ & $4.20 \mathrm{E}+00$ & $186 \mathrm{E} 05$ & $1.00 \mathrm{E}-01$ & $780 \mathrm{E}-04$ \\
\hline Zinc-65 & $2.90 \mathrm{E}+00$ & $4.35 \mathrm{E}-06$ & $2.40 \mathrm{E}+02$ & $1,81 \mathrm{E}-08$ & $1.00 \mathrm{E}+01$ & $435 \mathrm{E}-07$ \\
\hline Zirconium-93 & $1.00 \mathrm{E}+00$ & $1.50 \mathrm{E}-06$ & $6.20 \mathrm{E}+01$ & $242 \mathrm{E}-08$ & $1.00 \mathrm{E}+00$ & $1.50 \mathrm{E}-06$ \\
\hline Zirconium-95 & $3.10 \mathrm{E}+00$ & $4.65 \mathrm{E}-06$ & $7.00 \mathrm{E}+02$ & $6.64 \mathrm{E}-09$ & $1.00 \mathrm{E}+01$ & $4.65 \mathrm{E}-07$ \\
\hline Yttrium-90 & $1.10 \mathrm{E}+00$ & $1.65 \mathrm{E}-06$ & $1.42 \mathrm{E}+03$ & $1.16 \mathrm{E}-09$ & $1.00 \mathrm{E}+01$ & $1.65 \mathrm{E}-07$ \\
\hline \multicolumn{6}{|c|}{ Sum or the ratios of assumed concentrations to STD-1027 quantities $\times 5 \mathrm{E}-03^{\mathrm{a}}$} & \multirow[b]{2}{*}{$7 \mathrm{E}-03$} \\
\hline \multicolumn{6}{|c|}{ Sum of the ratios of assumed concentrations to 40 CFR 302 Appendix B releasable quantities $x$ EE- $03^{a}$} & \\
\hline \multicolumn{7}{|c|}{$\begin{array}{l}\text { Notes: } \\
\text { a The factor } 5 \mathrm{E}-03 \text { is the ratio of the release fraction of } 1.0 \mathrm{E}-03 \text { inherent in the TQs and RQs to the release fraction of } 5.0 \mathrm{E}-06 \text { (per tele- } \\
\text { con. with } \mathrm{H} \text {. Goldin regarding Draft EM Hazard Categorization Standard). } \\
\text { ND = No sample data. }\end{array}$} \\
\hline
\end{tabular}


Table A-2. Values for radionuclides in water.

\begin{tabular}{|c|c|c|c|c|c|c|}
\hline Contaminant & $\begin{array}{l}\text { Assumed water } \\
\text { concentration } \\
(\mathrm{pCi} / \mathrm{L})\end{array}$ & $\begin{array}{l}\text { Assumed } \\
\text { quantity } \\
\text { (Ci) } \\
\end{array}$ & $\begin{array}{c}\text { Category } 3 \\
\text { TQs } \\
\text { (Ci) } \\
\end{array}$ & $\begin{array}{l}\text { Ratio of assumed } \\
\text { guantity to the } \mathrm{PQ}\end{array}$ & $\begin{array}{l}\text { RQs } \\
\text { (Ci) } \\
\end{array}$ & $\begin{array}{l}\text { Ratio of } \\
\text { assumed } \\
\text { quantity to } \\
\text { the RQ }\end{array}$ \\
\hline Actinium-228 & ND & NA & $1.00 E+01$ & $\mathrm{NA}$ & $1.00 \mathrm{E}+01$ & $\mathrm{NA}$ \\
\hline Americium-241 & $1.70 \mathrm{E}+04$ & $6.80 \mathrm{E}-05$ & $1.00 \mathrm{E}+01$ & $680 \mathrm{E}-06$ & $1.00 \mathrm{E}-02$ & $6.80 \mathrm{E}-03$ \\
\hline Americium-242 & $1.30 \mathrm{E}+02$ & $5.20 \mathrm{E}-07$ & $8.20 \mathrm{E}+03$ & $634 \mathrm{E}-1$ & $1.00 \mathrm{E}+02$ & $520 \mathrm{E}-09$ \\
\hline Antimony-124 & $1.50 \mathrm{E}+02$ & $6.00 \mathrm{E}-07$ & $3.60 \mathrm{E}+02$ & $167 \mathrm{E} 09$ & $1.00 \mathrm{E}+01$ & $600 \mathrm{E}-08$ \\
\hline Californium-252 & ND & NA & $3.20 \mathrm{E}+00$ & $\mathrm{NA}$ & $1.00 \mathrm{E}-0 \mathrm{I}$ & $\mathrm{NA}$ \\
\hline Cerium-141 & $6.10 \mathrm{E}+03$ & $2.44 \mathrm{E}-05$ & $1.00 \mathrm{E}+03$ & $244 \mathrm{E}-08$ & $1.00 \mathrm{E}+01$ & $244 \mathrm{E}-06$ \\
\hline Cerium-144 & ND & NA & $1.00 \mathrm{E}+02$ & $\mathrm{NA}$ & $1.00 \mathrm{E}+00$ & $\mathrm{NA}$ \\
\hline Cesium-134 & $6.20 \mathrm{E}+04$ & $2.48 \mathrm{E}-04$ & $1.54 \mathrm{E}+03$ & $161 E-07$ & $1.00 \mathrm{E}+00$ & $2.48 \mathrm{E}-04$ \\
\hline Cesium-137 & $2.50 \mathrm{E}+08$ & $1.00 \mathrm{E}+00$ & $1.38 \mathrm{E}+05$ & $725 \mathrm{E}-06$ & $1.00 \mathrm{E}+00$ & $100 \mathrm{E}+00$ \\
\hline Chromium-51 & $2.50 \mathrm{E}+06$ & $1.00 \mathrm{E}-02$ & $2.20 \mathrm{E}+04$ & $4.55 \mathrm{E}-07$ & $1.00 \mathrm{E}+03$ & $100 \mathrm{E}-05$ \\
\hline Cobalt-5? & ND & NA & $6.00 \mathrm{E}+03$ & $\mathrm{NA}$ & $1.00 \mathrm{E}+02$ & $\mathrm{NA}$ \\
\hline Cobalt-58 & $6.00 \mathrm{E}+02$ & $2.40 \mathrm{E}-06$ & $9.00 \mathrm{E}+02$ & $2.67 \mathrm{E} 09$ & $1.00 \mathrm{E}+01$ & $2.40 \mathrm{E}-07$ \\
\hline Cobalt-60 & $1.20 \mathrm{E}+07$ & $4.80 \mathrm{E}-02$ & $2.80 \mathrm{E}+02$ & $1,71 \mathrm{E}-04$ & $1.00 \mathrm{E}+01$ & $480 \mathrm{E}-03$ \\
\hline Curium-242 & ND & NA & $3.20 \mathrm{E}+01$ & $\mathrm{NA}$ & $1.00 \mathrm{E}+00$ & $\mathrm{NA}$ \\
\hline Curium-243 & ND & NA & $8.20 \mathrm{E}+00$ & $\mathrm{NA}$ & $1.00 \mathrm{E}-02$ & $\mathrm{NA}$ \\
\hline Curium-244 & $1.60 \mathrm{E}+02$ & $6.40 \mathrm{E}-07$ & $1.05 \mathrm{E}+00$ & $610 \mathrm{E}-07$ & $1.00 \mathrm{E}-02$ & $6.40 \mathrm{E}-05$ \\
\hline Europium-152 & $1.10 \mathrm{E}+05$ & $4.40 \mathrm{E}-04$ & $2.00 \mathrm{E}+02$ & $220 \mathrm{E}-06$ & $1.00 \mathrm{E}+01$ & $4.40 \mathrm{E}_{-} 05$ \\
\hline Europium-154 & $1.30 E+05$ & $5.20 \mathrm{E}-04$ & $2.00 \mathrm{E}+02$ & $260 \mathrm{E}-06$ & $1.00 \mathrm{E}+01$ & $520 \mathrm{E}-05$ \\
\hline Europium-155 & $2.00 \mathrm{E}+04$ & $8.00 \mathrm{E}-05$ & $2.00 E+02$ & $400 \mathrm{E}-07$ & $1.00 \mathrm{E}+01$ & $800 \mathrm{E}-06$ \\
\hline Hafnium-175 & $3.50 \mathrm{E}+03$ & $1.40 \mathrm{E}-05$ & $2.00 \mathrm{E}+03$ & $100 \mathrm{E}-09$ & $1.00 \mathrm{E}+02$ & $140 \mathrm{E}-07$ \\
\hline Hafnium-181 & $1.40 \mathrm{E}+05$ & $5.60 \mathrm{E}-04$ & $7.60 \mathrm{E}+02$ & 7371007 & $1.00 \mathrm{E}+01$ & $560 \mathrm{E}-05$ \\
\hline Iodine-129 & ND & NA & $6.00 \mathrm{E}-02$ & $\mathrm{NA}$ & $1.00 \mathrm{E}-03$ & $\mathrm{NA}$ \\
\hline Iron-59 & $2.60 \mathrm{E}+03$ & $1.04 \mathrm{E}-05$ & $6.00 \mathrm{E}+02$ & $173 \mathrm{E}-08$ & $1.00 \mathrm{E}+01$ & $104 \mathrm{E}-06$ \\
\hline Lead-214 & ND & NA & $8.20 \mathrm{E}+03$ & $\mathrm{NA}$ & $1.00 \mathrm{E}+02$ & $\mathrm{NA}$ \\
\hline Manganese 54 & $3.40 \mathrm{E}+02$ & $1.36 \mathrm{E}-06$ & $8.80 \mathrm{E}+02$ & $155 \mathrm{E}-09$ & $1.00 \mathrm{E}+01$ & $136 \mathrm{E}-07$ \\
\hline Mercury-203 & $1.70 \mathrm{E}+03$ & $6.80 \mathrm{E}-06$ & $3.60 \mathrm{E}+02$ & $189 \mathrm{E}-08$ & $1.00 \mathrm{E}+01$ & $680 \mathrm{E}-07$ \\
\hline Neptunium-137 & $2.20 \mathrm{E}+02$ & $8.80 \mathrm{E}-07$ & $4.20 \mathrm{E}-01$ & $210 \mathrm{E}-06$ & $1.00 \mathrm{E}-02$ & $880 \mathrm{E}-05$ \\
\hline Niobium-94 & ND & NA & $2.00 \mathrm{E}+02$ & $\mathrm{NA}$ & $1.00 \mathrm{E}+01$ & $\mathrm{NA}$ \\
\hline Niobium-95 & $1.20 \mathrm{E}+04$ & $4.80 \mathrm{E}-05$ & $9.60 \mathrm{E}+02$ & 500008 & $1.00 \mathrm{E}+01$ & $4.80 \mathrm{E}-06$ \\
\hline Niobium-97 & ND & NA & $1.48 \mathrm{E}+03$ & $\mathrm{NA}$ & $1.00 \mathrm{E}+01$ & $\mathrm{NA}$ \\
\hline Plutonium-238 & $1.00 \mathrm{E}+05$ & $4.00 \mathrm{E}-04$ & $6.20 \mathrm{E}-01$ & $645 \mathrm{E} 04$ & $1.00 \mathrm{E}-02$ & $4.00 \mathrm{E}-02$ \\
\hline Plutonium-239/240 & $1.00 \mathrm{E}+05$ & $4.00 \mathrm{E}-04$ & $5.20 \mathrm{E}-01$ & 1.69E-04 & $1.00 \mathrm{E}-02$ & $400 \mathrm{E}-02$ \\
\hline Potassium-40 & ND & NA & $1.70 \mathrm{E}+02$ & $\mathrm{NA}$ & $1.00 \mathrm{E}+00$ & $\mathrm{NA}$ \\
\hline Radium-226 & ND & NA & $1.20 \mathrm{E}+01$ & $\mathrm{NA}$ & $1.00 \mathrm{E}-01$ & $\mathrm{NA}$ \\
\hline Radon-222 & $2.10 \mathrm{E}+02$ & $8.40 \mathrm{E}-07$ & $1.00 \mathrm{E}+01$ & $840 \mathrm{E}-08$ & $1.00 \mathrm{E}-01$ & $840 \mathrm{E}-06$ \\
\hline Rhodium-106m & $4.80 \mathrm{E}+03$ & $1.92 \mathrm{E}-05$ & $1.96 \mathrm{E}+03$ & $980 \mathrm{E}-09$ & $1.00 \mathrm{E}+01$ & $192 \mathrm{E}-06$ \\
\hline Ruthenium-103 & $4.00 E+03$ & $1.60 \mathrm{E}-05$ & $1.56 \mathrm{E}+03$ & $103 \mathrm{E}-08$ & $1.00 \mathrm{E}+01$ & $1.60 \mathrm{E}-06$ \\
\hline Scandium-46 & $4.10 \mathrm{E}+03$ & $1.64 \mathrm{E}-05$ & $3.60 \mathrm{E}+02$ & $456 \mathrm{E} 08$ & $1.00 \mathrm{E}+01$ & $164 \mathrm{E}-06$ \\
\hline Silver-108m & $1.40 \mathrm{E}+04$ & $5.60 \mathrm{E}-05$ & $2.00 \mathrm{E}+02$ & $280 \mathrm{E}-07$ & $1.00 \mathrm{E}+01$ & $560 \mathrm{E}-06$ \\
\hline Silver-110m & ND & NA & $2.60 \mathrm{E}+02$ & $\mathrm{NA}$ & $1.00 \mathrm{E}+01$ & $\mathrm{NA}$ \\
\hline Strontium-90 & $1.70 \mathrm{E}+08$ & $6.80 \mathrm{E}-01$ & $1.60 \mathrm{E}+01$ & $425 \mathrm{E}-02$ & $1.00 \mathrm{E}-01$ & $6,80 \mathrm{E}+00$ \\
\hline Tantalum-182 & $3.20 \mathrm{E}+03$ & $1.28 \mathrm{E}-05$ & $6.20 \mathrm{E}+02$ & $206 \mathrm{E}-08$ & $1.00 \mathrm{E}+02$ & $128 \mathrm{E}-07$ \\
\hline Thorium-228 & ND & NA & $1.00 \mathrm{E}+00$ & $\mathrm{NA}$ & $1.00 \mathrm{E}-02$ & $\mathrm{NA}$ \\
\hline Thorium-230 & ND & NA & $6.20 \mathrm{E}-01$ & $\mathrm{NA}$ & $1.00 \mathrm{E}-02$ & $\mathrm{NA}$ \\
\hline Thorium-232 & ND & NA & $1.00 \mathrm{E}-01$ & $\mathrm{NA}$ & $1.00 \mathrm{E}-03$ & $\mathrm{NA}$ \\
\hline Thorium-234 & $1.60 \mathrm{E}+01$ & $6.40 \mathrm{E}-08$ & $2.80 \mathrm{E}+03$ & $2.29 \mathrm{E}-11$ & $1.00 \mathrm{E}+02$ & $6.40 \mathrm{E}-10$ \\
\hline Tritium-3 & $4.30 \mathrm{E}+04$ & $1.72 \mathrm{E}-04$ & $1.00 \mathrm{E}+03$ & $172 \mathrm{E}-07$ & $1.00 \mathrm{E}+02$ & $172 \mathrm{E}-06$ \\
\hline
\end{tabular}


Table A-2. (continued).

\begin{tabular}{|c|c|c|c|c|c|c|}
\hline Contaminant & $\begin{array}{l}\text { Assumed water } \\
\text { concentration } \\
(\mathrm{pCi} / \mathrm{L})\end{array}$ & $\begin{array}{l}\text { Assumed } \\
\text { quantity } \\
\text { (Ci) }\end{array}$ & $\begin{array}{c}\text { Category } 3 \\
\text { TQs } \\
\text { (Ci) } \\
\end{array}$ & Ratio of assumed & $\begin{array}{l}\text { RQs } \\
\text { (Ci) }\end{array}$ & $\begin{array}{l}\text { Ratio of } \\
\text { assumed } \\
\text { quantity to } \\
\text { the RQ }\end{array}$ \\
\hline Uranium-232 & ND & $\mathrm{NA}$ & $8.20 \mathrm{E}-01$ & $\mathrm{NA}$ & $1.00 \mathrm{E}-02$ & NA \\
\hline Uranium-233 & ND & NA & $4.20 \mathrm{E}+00$ & $\mathrm{NA}$ & $1.00 \mathrm{E}-01$ & $\mathrm{NA}$ \\
\hline Uranium-234 & $9.00 \mathrm{E}+00$ & $3.60 \mathrm{E}-08$ & $4.20 \mathrm{E}+00$ & $857 \mathrm{H} 09$ & $1.00 \mathrm{E}-01$ & $3.60 \mathrm{E}-07$ \\
\hline Uranium-235 & $3.80 \mathrm{E}+00$ & $1.52 \mathrm{E}-08$ & $4.20 \mathrm{E}+00$ & $362 \mathrm{E}-09$ & $1.00 \mathrm{E}-01$ & $152 \mathrm{E}-07$ \\
\hline Uranium-238 & $2.40 \mathrm{E}+01$ & $9.60 \mathrm{E}-08$ & $4.20 \mathrm{E}+00$ & $229 \mathrm{E}-08$ & $1.00 \mathrm{E}-01$ & $9.60 \mathrm{E} \odot 07$ \\
\hline Zinc-65 & $1.10 \mathrm{E}+05$ & $4.40 \mathrm{E}-04$ & $2.40 \mathrm{E}+02$ & $183 \mathrm{E} 06$ & $1.00 \mathrm{E}+01$ & $4.40 \mathrm{E} 0 \mathrm{~S}$ \\
\hline Zirconium-95 & $1.20 \mathrm{E}+04$ & $4.80 \mathrm{E}-05$ & $7.00 \mathrm{E}+02$ & $686 \mathrm{E}-08$ & $1.00 \mathrm{E}+01$ & $480 \mathrm{E}-06$ \\
\hline \multicolumn{7}{|c|}{ Sum of the ratios of the assumed concentrations to 1027 quantities $\times 5 \mathrm{E}-03^{\mathrm{a}}$} \\
\hline \multicolumn{6}{|c|}{ Sum of the ratios of the assumed concentrations to the 40 CFR 302 releasable quantities $\times 5 E-03^{a}$} & $4 \mathrm{E}-02$ \\
\hline \multicolumn{7}{|l|}{ Notes: } \\
\hline \multicolumn{7}{|c|}{$\begin{array}{l}\text { a. The factor } 5 \mathrm{E}-03 \text { is the ratio of the release fraction of } 1.0 \mathrm{E}-03 \text { inherent in the TQs and RQs to the release fraction of } 5.0 \mathrm{E}-06 \text { (per tele- } \\
\text { con. with } \mathrm{H} \text {. Goldin regarding Draft EM Hazard Categorization Standard). } \\
\mathrm{ND}=\text { No sample data. }\end{array}$} \\
\hline \multicolumn{7}{|c|}{ NA = The calculation could not be performed because there are no sample data. } \\
\hline
\end{tabular}


Table A-3. Values for hazardous chemicals in soil or waste.

\begin{tabular}{|c|c|c|c|c|}
\hline Contaminant & $\begin{array}{l}\text { Assumed concentration } \\
(\mathrm{mg} / \mathrm{kg})\end{array}$ & $\begin{array}{c}\text { Assumed quantity } \\
(\mathrm{kg}) \\
\end{array}$ & $\begin{array}{l}\text { RQs } \\
(\mathrm{kg}) \\
\end{array}$ & Ratio of assumed quantity to the \\
\hline Acenaphthene & $9.60 \mathrm{E}-02$ & $1.44 \mathrm{E}-04$ & $4.54 \mathrm{E}+01$ & $317 \mathrm{E} 06$ \\
\hline Acetone & $1.30 \mathrm{E}+03$ & $1.95 \mathrm{E}+00$ & $2.27 \mathrm{E}+03$ & $859 \mathrm{E}-04$ \\
\hline Acrylonitrile & $4.70 \mathrm{E}+01$ & $7.05 \mathrm{E}-02$ & $4.54 \mathrm{E}+01$ & $155 \mathrm{E}-03$ \\
\hline Ammonium hydroxide & $9.00 \mathrm{E}+03$ & $1.35 \mathrm{E}+01$ & $4.54 \mathrm{E}+02$ & $297 \mathrm{E} 02$ \\
\hline Anthracene & $2.40 \mathrm{E}+01$ & $3.60 \mathrm{E}-02$ & $2.27 \mathrm{E}+03$ & $159 \mathrm{E}-05$ \\
\hline Antimony & $2.40 \mathrm{E}+03$ & $3.60 \mathrm{E}+00$ & $2.27 \mathrm{E}+03$ & $159 \mathrm{E}-03$ \\
\hline Arsenic & $2.10 \mathrm{E}+02$ & $3.15 \mathrm{E}-01$ & $4.54 \mathrm{E}-01$ & $694 \mathrm{E}-01$ \\
\hline Barium & $9.70 \mathrm{E}+03$ & $1.46 \mathrm{E}+01$ & $4.54 \mathrm{E}+00$ & $320 \mathrm{E}+00$ \\
\hline Benzene & $9.80 \mathrm{E}+02$ & $1.47 \mathrm{E}+00$ & $4.54 \mathrm{E}+00$ & $324 \mathrm{E}-0 \mathrm{I}$ \\
\hline Benzo(a)anthracene & $8.80 \mathrm{E}+01$ & $1.32 \mathrm{E}-01$ & $4.54 \mathrm{E}+00$ & $291 \mathrm{E}-02$ \\
\hline Benzo(a)pyrene & $5.60 \mathrm{E}+01$ & $8.40 \mathrm{E}-02$ & $4.54 \mathrm{E}-01$ & $185 \mathrm{E} 01$ \\
\hline Benzo(b)fluoranthene & $8.10 \mathrm{E}+01$ & $1.22 \mathrm{E}-01$ & $2.27 \mathrm{E}+03$ & $535 \mathrm{P} 05$ \\
\hline Benzo(g,h,i)perylene & $1.90 \mathrm{E}+01$ & $2.85 \mathrm{E}-02$ & $2.27 \mathrm{E}+03$ & $126 \mathrm{E} 05$ \\
\hline Benzoic acid & $3.20 \mathrm{E}-01$ & $4.80 \mathrm{E}-04$ & $2.27 \mathrm{E}+03$ & $211 \mathrm{E}-97$ \\
\hline Benzo(k)fluoranthene & $1.20 \mathrm{E}+00$ & $1.80 \mathrm{E}-03$ & $2.27 \mathrm{E}+03$ & $7: 93 \mathrm{E} 007$ \\
\hline Beryllium & $2.60 E+03$ & $3.90 \mathrm{E}+00$ & $4.54 \mathrm{E}+00$ & $859 \mathrm{E}-01$ \\
\hline Bis(2-chloroethyl)ether & $6.70 \mathrm{E}-02$ & $1.01 \mathrm{E}-04$ & $4.54 \mathrm{E}+00$ & $221 \mathrm{E} 05$ \\
\hline Bis(2-Ethylhexyl)Phthalate & $3.60 \mathrm{E}+02$ & $5.40 \mathrm{E}-01$ & $4.54 \mathrm{E}+01$ & $1,19 \mathrm{E}-02$ \\
\hline 2-butanone (MEK) & $1.80 \mathrm{E}+03$ & $2.70 \mathrm{E}+00$ & $2.27 \mathrm{E}+03$ & $1.19 \mathrm{E} 03$ \\
\hline Butyl benzylphthalate & $2.10 \mathrm{E}+01$ & $3.15 \mathrm{E}-02$ & $4.54 \mathrm{E}+01$ & $694 \mathrm{~B}-04$ \\
\hline Cadmium & $3.30 E+04$ & $4.95 \mathrm{E}+01$ & $4.54 \mathrm{E}+00$ & $109 \mathrm{E}+01$ \\
\hline Carbon disulfide & $2.30 \mathrm{E}+00$ & $3.45 \mathrm{E}-03$ & $4.54 \mathrm{E}+01$ & $760 \mathrm{E}-05$ \\
\hline Carbon tetrachloride & $5.10 \mathrm{E}+01$ & $7.65 \mathrm{E}-02$ & $4.54 \mathrm{E}+00$ & $1.69 \mathrm{E}-02$ \\
\hline 4-Chloroaniline & $1.10 \mathrm{E}+00$ & $1.65 \mathrm{E}-03$ & $4.54 \mathrm{E}+02$ & $363 \mathrm{E}-06$ \\
\hline Chlorobenzene & $9.20 \mathrm{E}+01$ & $1.38 \mathrm{E}-01$ & $4.54 \mathrm{E}+01$ & $304 \mathrm{E}-03$ \\
\hline Chloroethane & $7.70 \mathrm{E}+02$ & $1.16 \mathrm{E}+00$ & $4.54 \mathrm{E}+01$ & $254 \mathrm{E}-02$ \\
\hline Chloroform & $2.40 \mathrm{E}+02$ & $3.60 \mathrm{E}-01$ & $4.54 \mathrm{E}+00$ & $793 \mathrm{E}-02$ \\
\hline Chromium & $4.10 \mathrm{E}+04$ & $6.15 \mathrm{E}+01$ & $2.27 \mathrm{E}+03$ & $271 E 02$ \\
\hline Chrysene & $7.10 \mathrm{E}+01$ & $1.07 \mathrm{E}-01$ & $4.54 \mathrm{E}+01$ & $235 \mathrm{E}-03$ \\
\hline Copper & $2.90 \mathrm{E}+03$ & $4.35 \mathrm{E}+00$ & $2.27 \mathrm{E}+03$ & $192 \mathrm{E}-03$ \\
\hline Cresol & $2.20 \mathrm{E}+02$ & $3.30 \mathrm{E}-01$ & $4.54 \mathrm{E}+02$ & $727 \mathrm{E}-04$ \\
\hline Cyanide & $3.10 \mathrm{E}+02$ & $4.65 \mathrm{E}-01$ & $4.54 \mathrm{E}+00$ & $102 \mathrm{E} 01$ \\
\hline $4,4^{\prime}-$ DDT & $6.00 \mathrm{E}-02$ & $9.00 \mathrm{E}-05$ & $4.54 \mathrm{E}-01$ & $198 \mathrm{E}-04$ \\
\hline Dibenzo(a,h)anthracene & $8.70 \mathrm{E}+00$ & $1.31 \mathrm{E}-02$ & $4.54 \mathrm{E}-01$ & $2.87 \mathrm{E} 02$ \\
\hline Dibutylphthalate & $6.80 \mathrm{E}-02$ & $1.02 \mathrm{E}-04$ & $4.54 \mathrm{E}+00$ & $225 \mathrm{E} 05$ \\
\hline Dichlorobenzene & $2.80 \mathrm{E}+01$ & $4.20 \mathrm{E}-02$ & $4.54 \mathrm{E}+01$ & $925 \mathrm{E}-04$ \\
\hline 1,2-dichlorobenzene & $4.30 \mathrm{E}+00$ & $6.45 \mathrm{E}-03$ & $4.54 \mathrm{E}+01$ & $142 \mathrm{E}-04$ \\
\hline 1,4-dichlorobenzene & $2.60 \mathrm{E}+04$ & $3.90 \mathrm{E}+01$ & $4.54 \mathrm{E}+01$ & $859 \mathrm{E}-01$ \\
\hline Dichlorodifluoromethane & $2.20 \mathrm{E}+02$ & $3.30 \mathrm{E}-01$ & $2.27 \mathrm{E}+03$ & $145 \mathrm{E} 04$ \\
\hline 1,1 dichloroethane & $2.60 \mathrm{E}+02$ & $3.90 \mathrm{E}-01$ & $4.54 \mathrm{E}+02$ & $859 \mathrm{E}-04$ \\
\hline 1,1-dichloroethylene & $2.60 \mathrm{E}+02$ & $3.90 \mathrm{E}-01$ & $4.54 \mathrm{E}+01$ & $859 \mathrm{E}-03$ \\
\hline 1,2-dichloroethylene & $4.10 \mathrm{E}+02$ & $6.15 \mathrm{E}-01$ & $4.54 \mathrm{E}+02$ & $135 \mathrm{E}-03$ \\
\hline Diethylphthalate & $1.90 \mathrm{E}+00$ & $2.85 \mathrm{E}-03$ & $4.54 \mathrm{E}+02$ & $628 \mathrm{E}-06$ \\
\hline 2,4-dimethylphenol & $3.30 \mathrm{E}+00$ & $4.95 \mathrm{E}-03$ & $4.54 \mathrm{E}+01$ & $109 \mathrm{E}-04$ \\
\hline Di(n-octyl)phthalate & $3.60 \mathrm{E}+02$ & $5.40 \mathrm{E}-01$ & $2.27 \mathrm{E}+03$ & $238 \mathrm{E}-04$ \\
\hline Disulfoton & $1.30 \mathrm{E}+02$ & $1.95 \mathrm{E}-01$ & $4.54 \mathrm{E}-01$ & $430 \mathrm{E}-01$ \\
\hline Ethylbenzene & $1.30 \mathrm{E}+03$ & $1.95 \mathrm{E}+00$ & $4.54 \mathrm{E}+02$ & $4.30 \mathrm{E}-03$ \\
\hline Fluoranthene & $1.50 \mathrm{E}+02$ & $2.25 \mathrm{E}-01$ & $4.54 \mathrm{E}+01$ & $496 \mathrm{E}-03$ \\
\hline
\end{tabular}


Table A-3. (continued).

\begin{tabular}{|c|c|c|c|c|}
\hline Contaminant & $\begin{array}{c}\text { Assumed concentration } \\
(\mathrm{mg} / \mathrm{kg})\end{array}$ & $\begin{array}{l}\text { Assumed quantity } \\
(\mathrm{kg})\end{array}$ & $\begin{array}{l}\text { RQs } \\
(\mathrm{kg})\end{array}$ & $\begin{array}{l}\text { Ratio of assumed quantity to the } \\
\text { RQS }\end{array}$ \\
\hline Fluorene & $3.30 \mathrm{E}+01$ & $4.95 \mathrm{E}-02$ & $2.27 \mathrm{E}+03$ & $218 \mathrm{E} 05$ \\
\hline Fluorine & $3.90 \mathrm{E}+00$ & $5.85 \mathrm{E}-03$ & $4.54 \mathrm{E}+00$ & $129 \mathrm{E}-03$ \\
\hline $\begin{array}{l}\text { Hexone or 4-Methyl } \\
\text { 2-pentanone }\end{array}$ & $8.30 \mathrm{E}+00$ & $1.25 \mathrm{E}-02$ & $2.27 \mathrm{E}+03$ & $5.48 \mathrm{E} 06$ \\
\hline Hydrazine & $1.80 \mathrm{E}-01$ & $2.70 \mathrm{E}-04$ & $4.54 \mathrm{E}-01$ & $595 \mathrm{E}-04$ \\
\hline Hydrochloric acid & $8.60 \mathrm{E}+02$ & $1.29 \mathrm{E}+00$ & $2.27 \mathrm{E}+03$ & $5,68 \mathrm{E} 04$ \\
\hline Ideno(1,2,3-cd)pyrene & $1.80 \mathrm{E}+01$ & $2.70 \mathrm{E}-02$ & $4.54 \mathrm{E}+01$ & $595 \mathrm{E}-04$ \\
\hline Isophorone & $8.50 \mathrm{E}+01$ & $1.28 \mathrm{E}-0 \mathrm{I}$ & $2.27 E+03$ & $562 t-05$ \\
\hline Kepone & $7.00 \mathrm{E}-02$ & $1.05 \mathrm{E}-04$ & $4.54 \mathrm{E}-01$ & $231 \mathrm{E}-04$ \\
\hline Lead & $2.90 \mathrm{E}+04$ & $4.35 \mathrm{E}+01$ & $4.54 \mathrm{E}+00$ & $958 \mathrm{E}+00$ \\
\hline Mercury & $4.00 \mathrm{E}+03$ & $6.00 \mathrm{E}+00$ & $4.54 \mathrm{E}-01$ & $132 \mathrm{E}+01$ \\
\hline Methanol & $6.60 \mathrm{E}+02$ & $9.90 \mathrm{E}-01$ & $2.27 \mathrm{E}+03$ & $436 \mathrm{E} 04$ \\
\hline Methylene chloride & $1.10 \mathrm{E}+03$ & $1.65 \mathrm{E}+00$ & $4.54 \mathrm{E}+02$ & $3,63 \mathrm{E} 03$ \\
\hline Naphthalene & $6.80 \mathrm{E}+01$ & $1.02 \mathrm{E}-01$ & $4.54 \mathrm{E}+01$ & $225 \mathrm{E}-13$ \\
\hline Nickel & $5.90 \mathrm{E}+03$ & $8.85 E+00$ & $4.54 \mathrm{E}+01$ & $195 \mathrm{E}-01$ \\
\hline Nitric acid & $2.90 \mathrm{E}+03$ & $4.35 \mathrm{E}+00$ & $4.54 \mathrm{E}+02$ & $958 \mathrm{E}-03$ \\
\hline Nitrobenzene & $5.00 \mathrm{E}-03$ & $7.50 \mathrm{E}-06$ & $4.54 \mathrm{E}+02$ & $165 \mathrm{E}-08$ \\
\hline 2-nitrophenol & $1.00 \mathrm{E}-03$ & $1.50 \mathrm{E}-06$ & $4.54 \mathrm{E}+01$ & $330 \mathrm{E} 08$ \\
\hline 4-nitrophenol & $2.60 \mathrm{E}-01$ & $3.90 \mathrm{E}-04$ & $4.54 \mathrm{E}+01$ & $8.59 \mathrm{E}-06$ \\
\hline $\mathrm{N}$-nitrosodiphenylamine & $3.00 \mathrm{E}-02$ & $4.50 \mathrm{E}-05$ & $4.54 \mathrm{E}+01$ & $991 \mathrm{E}-07$ \\
\hline PCB(Aroclor 1016) & $1.30 \mathrm{E}+00$ & $1.95 \mathrm{E}-03$ & $4.54 \mathrm{E}-01$ & $430 \mathrm{E}-03$ \\
\hline PCB (Arclor 1242) & $3.80 \mathrm{E}+02$ & $5.70 \mathrm{E}-01$ & $4.54 \mathrm{E}-01$ & $126 \mathrm{E}+00$ \\
\hline PCB (Arclor 1248) & $2.40 \mathrm{E}-02$ & $3.60 \mathrm{E}-05$ & $4.54 \mathrm{E}-01$ & $193 \mathrm{E}-05$ \\
\hline PCB (Arclor 1254) & $5.20 \mathrm{E}+02$ & $7.80 \mathrm{E}-01$ & $4.54 \mathrm{E}-01$ & $172 \mathrm{E}+00$ \\
\hline PCB (Aroclor 1260) & $7.10 \mathrm{E}+03$ & $1.07 \mathrm{E}+01$ & $4.54 \mathrm{E}-01$ & $235 \mathrm{E}+01$ \\
\hline Pentachlorophenol & $3.80 \mathrm{E}-01$ & $5.70 \mathrm{E}-04$ & $4.54 \mathrm{E}+00$ & $126 \mathrm{E}-04$ \\
\hline Phenanthrene & $2.30 \mathrm{E}+02$ & $3.45 \mathrm{E}-01$ & $2.27 \mathrm{E}+03$ & $152 \mathrm{E}-04$ \\
\hline Phenol & $7.00 E+02$ & $1.05 E+00$ & $4.54 \mathrm{E}+02$ & $231 \mathrm{E}-03$ \\
\hline Potassium & $8.60 \mathrm{E} \div 03$ & $1.29 \mathrm{E}+01$ & $4.54 \mathrm{E}-01$ & $284 \mathrm{E}+01$ \\
\hline Potassium chromate & $2.30 \mathrm{E}+03$ & $3.45 \mathrm{E}+00$ & $4.54 \mathrm{E}+00$ & $760 \mathrm{E}-01$ \\
\hline Pyrene & $2.10 \mathrm{E}+02$ & $3.15 \mathrm{E}-01$ & $2.27 E+03$ & $139 \mathrm{E}-4$ \\
\hline Selenium & $1.30 \mathrm{E}+02$ & $1.95 \mathrm{E}-01$ & $4.54 \mathrm{E}+01$ & $430 \mathrm{E}-03$ \\
\hline Sodium & $6.30 \mathrm{E}+03$ & $9.45 \mathrm{E}+00$ & $4.54 \mathrm{E}+00$ & $208 \mathrm{E}+00$ \\
\hline Strontium & $6.00 \mathrm{E}+02$ & $9.00 \mathrm{E}-01$ & $4.54 \mathrm{E}+00$ & $198 \mathrm{E} 01$ \\
\hline Sulfuric acid & $3.60 \mathrm{E}+05$ & $5.40 \mathrm{E}+02$ & $4.54 \mathrm{E}+02$ & $119 \mathrm{E}+00$ \\
\hline 2,4,5-TP(Silvex) & $1.60 \mathrm{E}-01$ & $2.40 \mathrm{E}-04$ & $4.54 \mathrm{E}+01$ & $529 \mathrm{E}-06$ \\
\hline 1,1,2,2-tetrachloroethane & $3.00 \mathrm{E}-01$ & $4.50 \mathrm{E}-04$ & $4.54 \mathrm{E}+01$ & $991 \mathrm{E}-06$ \\
\hline Tetrachloroethene & $2.80 E+03$ & $4.20 \mathrm{E}+00$ & $4.54 \mathrm{E}+01$ & $9.25 \mathrm{E}-02$ \\
\hline Tetrahydrofuran & $2.50 \mathrm{E}-02$ & $3.75 \mathrm{E}-05$ & $4.54 \mathrm{E}+02$ & $826 \mathrm{E}-08$ \\
\hline Thallium & $4.80 \mathrm{E}+0 \mathrm{I}$ & $7.20 \mathrm{E}-02$ & $4.54 \mathrm{E}+02$ & $159 \mathrm{E}-04$ \\
\hline Toluene & $1.20 \mathrm{E}+04$ & $1.80 \mathrm{E}+01$ & $4.54 \mathrm{E}+02$ & $396 \mathrm{E} 02$ \\
\hline 2,4,5-TP (Silvex) & $1.80 \mathrm{E}-01$ & $2.70 \mathrm{E}-04$ & $4.54 \mathrm{E}+01$ & $595 \mathrm{E}-06$ \\
\hline $1,1,1$ trichloroethane & $2.10 \mathrm{E}+03$ & $3.15 \mathrm{E}+00$ & $4.54 \mathrm{E}+02$ & $694 \mathrm{E}-03$ \\
\hline 1,1,2-trichloroethane & $1.00 \mathrm{E}-03$ & $1.50 \mathrm{E}-06$ & $4.54 \mathrm{E}+01$ & $3,30 \mathrm{E}-8$ \\
\hline Trichloroethylene & $3.00 \mathrm{E}+04$ & $4.50 \mathrm{E}+01$ & $4.54 \mathrm{E}+01$ & $991 \mathrm{E}-01$ \\
\hline Vanadium & $4.80 \mathrm{E}+02$ & $7.20 \mathrm{E}-01$ & $4.54 \mathrm{E}+02$ & $1,59 \mathrm{E}-03$ \\
\hline Vinyl acetate & $3.00 \mathrm{E}-03$ & $4.50 \mathrm{E}-06$ & $2.27 \mathrm{E}+03$ & $198 \mathrm{E}-09$ \\
\hline Vinyl chloride & $6.30 \mathrm{E}-01$ & $9.45 \mathrm{E}-04$ & $4.54 \mathrm{E}-01$ & $2.08 \mathrm{E}-03$ \\
\hline
\end{tabular}


Table A-3. (continued).

\begin{tabular}{|c|c|c|c|c|}
\hline Contaminant & $\begin{array}{c}\text { Assumed concentration } \\
(\mathrm{mg} / \mathrm{kg})\end{array}$ & $\begin{array}{l}\text { Assumed quantity } \\
(\mathrm{kg})\end{array}$ & $\begin{array}{l}\mathrm{RQs} \\
(\mathrm{kg})\end{array}$ & $\begin{array}{l}\text { Ratio of assumed quantity to the } \\
\text { RQs }\end{array}$ \\
\hline Xylene (total) & $3.00 \mathrm{E}+04$ & $4.50 \mathrm{E}+01$ & $4.54 \mathrm{E}+02$ & $991 \mathrm{E}-02$ \\
\hline Zinc & $1.60 \mathrm{E}+04$ & $2.40 \mathrm{E}+01$ & $4.54 \mathrm{E}+02$ & $529 \mathrm{E}-02$ \\
\hline \multicolumn{4}{|c|}{ Sum of the ratios of the releasable quantities $\times 5 \mathrm{E}-3^{2}$} & $5 \mathrm{E}-01$ \\
\hline
\end{tabular}

Notes:

a. The factor $5 \mathrm{E}-03$ is the ratio of the release fraction of $1.0 \mathrm{E}-03$ inherent in the TQs and RQs to the release fraction of $5.0 \mathrm{E}-06$ (per telecon with $\mathrm{H}$. Goldin regariding Draft EM Hazard Categorization Standard).

Table A-4. Values for hazardous chemicals in water.

\begin{tabular}{|c|c|c|c|c|}
\hline Contaminant & $\begin{array}{l}\text { Assumed concentration } \\
(\mu \mathrm{g} / \mathrm{L})\end{array}$ & $\begin{array}{l}\text { Assumed quantity } \\
(\mathrm{kg})\end{array}$ & $\begin{array}{l}\text { RQs } \\
(\mathrm{kg})\end{array}$ & Ratio of assumed quantity to the RQs \\
\hline Acetone & $7.20 \mathrm{E}+04$ & $2.88 \mathrm{E}-0 \mathrm{l}$ & $2.27 \mathrm{E}+03$ & $130 \mathrm{E}-04$ \\
\hline Aluminum & $4.30 \mathrm{E}+05$ & $1.72 \mathrm{E}+00$ & $4.54 \mathrm{E}+01$ & $380 \mathrm{E}-02$ \\
\hline Antimony & $1.40 \mathrm{E}+02$ & $5.60 \mathrm{E}-04$ & $2.27 \mathrm{E}+03$ & $250 \mathrm{E}-07$ \\
\hline Arsenic & $3.60 \mathrm{E}+04$ & $1.44 \mathrm{E}-01$ & $4.54 \mathrm{E}-01$ & $320 \mathrm{E} \odot 01$ \\
\hline Barium & $1.00 \mathrm{E}+04$ & $4.00 \mathrm{E}-02$ & $4.54 \mathrm{E}+00$ & $880 \mathrm{E}-03$ \\
\hline Benzene & $2.00 \mathrm{E}+00$ & $8.00 \mathrm{E}-06$ & $4.54 \mathrm{E}+00$ & $80 \mathrm{E}-06$ \\
\hline Benzo(a)anthracene & $5.50 \mathrm{E}-03$ & $2.20 \mathrm{E}-08$ & $4.54 \mathrm{E}+00$ & $480 \mathrm{E} 09$ \\
\hline Benzo(a)pyrene & $1.30 \mathrm{E}-03$ & $5.20 \mathrm{E}-09$ & $4.54 \mathrm{E}-01$ & $110 \mathrm{E}-08$ \\
\hline Benzo(b)fluoranthene & $3.30 \mathrm{E}-03$ & 1.32E-08 & $2.27 \mathrm{E}+03$ & $580 \mathrm{E}-12$ \\
\hline Benzo(g,h,i)perylene & $8.50 \mathrm{E}-04$ & $3.40 \mathrm{E}-09$ & $2.27 \mathrm{E}+03$ & $50 \mathrm{E} 12$ \\
\hline Benzo(k)fluoranthene & $3.30 \mathrm{E}-03$ & $1.32 \mathrm{E}-08$ & $2.27 \mathrm{E}+03$ & $380 \mathrm{E} 12$ \\
\hline Beryllium & $1.40 \mathrm{E}+02$ & $5.60 \mathrm{E}-04$ & $4.54 \mathrm{E}+00$ & $120 \mathrm{E}-04$ \\
\hline Bis(2-ethylhexyl)phthalate & $1.90 \mathrm{E}+02$ & $7.60 \mathrm{E}-04$ & $4.54 \mathrm{E}+01$ & $170 \mathrm{E} 005$ \\
\hline Bromoform & $1.00 \mathrm{E}+00$ & $4.00 \mathrm{E}-06$ & $4.54 \mathrm{E}+01$ & $880 \mathrm{E}-08$ \\
\hline 2-butanone (MEK) & $3.00 \mathrm{E}+04$ & $1.20 \mathrm{E}-01$ & $2.27 \mathrm{E}+03$ & $530 \mathrm{E} 05$ \\
\hline Butylbenzylphthalate & $6.00 \mathrm{E}+00$ & $2.40 \mathrm{E}-05$ & $4.54 \mathrm{E}+01$ & $530 \mathrm{E} 07$ \\
\hline Cadmium & $6.20 \mathrm{E}+03$ & $2.48 \mathrm{E}-02$ & $4.54 \mathrm{E}+00$ & $550 \mathrm{E}-03$ \\
\hline Calcium & $9.00 \mathrm{E}+05$ & $3.60 \mathrm{E}+00$ & $4.54 \mathrm{E}+00$ & $790 \mathrm{E}-01$ \\
\hline Carbon disulfide & $1.80 \mathrm{E}+01$ & $7.20 \mathrm{E}-05$ & $4.54 \mathrm{E}+01$ & $1.60 \mathrm{E}-06$ \\
\hline Carbon tetrachloride & $5.00 \mathrm{E}+00$ & $2.00 \mathrm{E}-05$ & $4.54 \mathrm{E}+00$ & $440 \mathrm{E}-06$ \\
\hline Chlorine & $2.00 \mathrm{E}+04$ & $8.00 \mathrm{E}-02$ & $4.54 \mathrm{E}+00$ & $180 \mathrm{E}-02$ \\
\hline Chlorobenzene & $1.60 \mathrm{E}+01$ & $6.40 \mathrm{E}-05$ & $4.54 \mathrm{E}+01$ & $140 \mathrm{E}-06$ \\
\hline Chloroform & $7.00 \mathrm{E}+00$ & $2.80 \mathrm{E}-05$ & $4.54 \mathrm{E}+00$ & $620 \mathrm{E}-06$ \\
\hline Chromium & $1.10 \mathrm{E}+04$ & $4.40 \mathrm{E}-02$ & $2.27 \mathrm{E}+03$ & $190 \mathrm{~B}-05$ \\
\hline Chrysene & $4.30 \mathrm{E}-02$ & $1.72 \mathrm{E}-07$ & $4.54 \mathrm{E}+01$ & $3,80 \mathrm{E} 00$ \\
\hline Copper & $1.90 \mathrm{E}+03$ & $7.60 \mathrm{E}-03$ & $2.27 \mathrm{E}+03$ & $3,30 \mathrm{E}=06$ \\
\hline Cyanide & $5.50 \mathrm{E}+00$ & $2.20 \mathrm{E}-05$ & $4.54 \mathrm{E}+00$ & $480 \mathrm{E}=06$ \\
\hline 1,2-dichlorobenzene & $1.90 \mathrm{E}+00$ & $7.60 \mathrm{E}-06$ & $4.54 \mathrm{E}+01$ & $170 \mathrm{E}-07$ \\
\hline 1,4-dichlorobenzene & $5.00 \mathrm{E}+00$ & $2.00 \mathrm{E}-05$ & $4.54 \mathrm{E}+01$ & $440 \mathrm{E}-07$ \\
\hline Dichlorodifluoromethane & $3.90 \mathrm{E}+03$ & $1.56 \mathrm{E}-02$ & $2.27 \mathrm{E}+03$ & $690 \mathrm{E}-06$ \\
\hline 1,1-dichloroethane & $1.10 \mathrm{E}+01$ & $4.40 \mathrm{E}-05$ & $4.54 \mathrm{E}+02$ & $970 \mathrm{E}-08$ \\
\hline
\end{tabular}


Table A-4. (continued).

\begin{tabular}{|c|c|c|c|c|}
\hline Contaminant & $\begin{array}{l}\text { Assumed concentration } \\
(\mu \mathrm{g} / \mathrm{L})\end{array}$ & $\begin{array}{l}\text { Assumed quantity } \\
(\mathrm{kg})\end{array}$ & $\begin{array}{l}\mathrm{RQs} \\
(\mathrm{kg})\end{array}$ & Ratio of assumed quantity to the RQs \\
\hline 1,2-dichloroethane & $8.50 \mathrm{E}+01$ & $3.40 \mathrm{E}-04$ & $4.54 \mathrm{E}+01$ & 750106 \\
\hline 1,1-dichloroethylene & $8.50 \mathrm{E}+01$ & $3.40 \mathrm{E}-04$ & $4.54 \mathrm{E}+01$ & $750 \mathrm{E}-06$ \\
\hline 1,2-dichloroethylene & $9.30 E+03$ & $3.72 \mathrm{E}-02$ & $4.54 \mathrm{E}+02$ & $820 \mathrm{E}-05$ \\
\hline Diethylphthalate & $1.00 \mathrm{E}+00$ & $4.00 \mathrm{E}-06$ & $4.54 \mathrm{E}+02$ & $880 \mathrm{E}-09$ \\
\hline Dimethylphthalate & $6.00 \mathrm{E}+00$ & $2.40 \mathrm{E}-05$ & $2.27+03$ & $110 \mathrm{E} 0 \mathrm{~S}$ \\
\hline Di(n-octyl)phthalate & $6.20 \mathrm{E}+01$ & $2.48 \mathrm{E}-04$ & $2.27 \mathrm{E}+03$ & $110 \mathrm{E}-07$ \\
\hline Ethylbenzene & $2.00 \mathrm{E}+03$ & $8.00 \mathrm{E}-03$ & $4.54 \mathrm{E}+02$ & $180 \mathrm{E} 05$ \\
\hline Fluoranthene & $2.20 \mathrm{E}-02$ & $8.80 \mathrm{E}-08$ & $4.54 \mathrm{E}+01$ & $190 \mathrm{E} 09$ \\
\hline $\begin{array}{l}\text { hexone same as 4-methyl } \\
\text { 2-pentanone }\end{array}$ & $3.20 \mathrm{E}+01$ & $1.28 \mathrm{E}-04$ & $2.27 \mathrm{E}+03$ & $5,60 \mathrm{E}-08$ \\
\hline Lead & $9.40 \mathrm{E}+03$ & $3.76 \mathrm{E}-02$ & $4.54 \mathrm{E}+00$ & $830 \mathrm{E}-03$ \\
\hline Mercury & $7.30 \mathrm{E}+04$ & $2.92 \mathrm{E}-01$ & $4.54 \mathrm{E}-01$ & $640 \mathrm{E} 01$ \\
\hline Methylene chloride & $3.40 \mathrm{E}+01$ & $1.36 \mathrm{E}-04$ & $4.54 \mathrm{E}+02$ & $300 \mathrm{E}-07$ \\
\hline Naphthalene & $8.00 \mathrm{E}-01$ & $3.20 \mathrm{E}-06$ & $4.54 \mathrm{E}+01$ & $700 \mathrm{E}-08$ \\
\hline Nickel & $6.70 E+03$ & $2.68 \mathrm{E}-02$ & $4.54 \mathrm{E}+01$ & $590 \mathrm{E}-04$ \\
\hline Phenol & $1.40 \mathrm{E}+01$ & $5.60 \mathrm{E}-05$ & $4.54 \mathrm{E}+02$ & $120 \mathrm{E}-07$ \\
\hline Pentachlorophenol & $1.60 \mathrm{E}-02$ & $6.40 \mathrm{E}-08$ & $4.54 \mathrm{E}+00$ & 140E-08 \\
\hline Phenanthrene & $1.30 \mathrm{E}-01$ & $5.20 \mathrm{E}-07$ & $2.27 \mathrm{E}+03$ & $230 \mathrm{E}-10$ \\
\hline Potassium & $4.70 \mathrm{E}+04$ & $1.88 \mathrm{E}-01$ & $4.54 \mathrm{E}-01$ & $410 \mathrm{E} 01$ \\
\hline Pyrene & $9.90 \mathrm{E}-02$ & $3.96 \mathrm{E}-07$ & $2.27 \mathrm{E}+03$ & $170 \mathrm{E}-10$ \\
\hline Selenium & $3.00 \mathrm{E}+02$ & $1.20 \mathrm{E}-03$ & $4.54 \mathrm{E}+01$ & $260 \mathrm{E} 05$ \\
\hline Silver & $3.20 \mathrm{E}+02$ & $1.28 \mathrm{E}-03$ & $4.54 \mathrm{E}+02$ & $2801-06$ \\
\hline Sodium & $2.10 \mathrm{E}+06$ & $8.40 \mathrm{E}+00$ & $4.54 \mathrm{E}+00$ & $190 \mathrm{E}+00$ \\
\hline Strontium & $1.10 \mathrm{E}+03$ & $4.40 \mathrm{E}-03$ & $4.54 \mathrm{E}+00$ & $970 \mathrm{E} 04$ \\
\hline Tetrachloroethene & $5.80 \mathrm{E}+02$ & $2.32 \mathrm{E}-03$ & $4.54 \mathrm{E}+01$ & $510 \mathrm{E}-05$ \\
\hline Thallium & $2.00 \mathrm{E}+02$ & $8.00 \mathrm{E}-04$ & $4.54 \mathrm{E}+02$ & $180 \mathrm{E}-06$ \\
\hline Toluene & $7.10 \mathrm{E}+02$ & $2.84 \mathrm{E}-03$ & $4.54 \mathrm{E}+02$ & $630 \mathrm{E} 06$ \\
\hline 1,2,4-trichlorobenzene & $4.00 \mathrm{E}-01$ & $1.60 \mathrm{E}-06$ & $4.54 \mathrm{E}+01$ & $350 \mathrm{~F}-08$ \\
\hline 1,1,1-trichloroethane & $1.80 \mathrm{E}+08$ & $7.20 \mathrm{E}+02$ & $4.54 \mathrm{E}+02$ & $160 \mathrm{E}+00$ \\
\hline 1,1,2-trichloroethane & $5.00 \mathrm{E}+00$ & $2.00 \mathrm{E}-05$ & $4.54 \mathrm{E}+01$ & $440 \mathrm{E}-07$ \\
\hline $\begin{array}{l}\text { Trichloroethene or Trichlo- } \\
\text { roethylene }\end{array}$ & $3.20 \mathrm{E}+04$ & $1.28 \mathrm{E}-01$ & $4.54 \mathrm{E}+01$ & $2.80 \mathrm{E}-03$ \\
\hline Vanadium & $7.60 \mathrm{E}+02$ & $3.04 \mathrm{E}-03$ & $4.54 \mathrm{E}+02$ & $6,70 \mathrm{E}-06$ \\
\hline Xylene (total) & $3.60 \mathrm{E}+03$ & $1.44 \mathrm{E}-02$ & $4.54 \mathrm{E}+02$ & $320 E 05$ \\
\hline Zine & $1.10 \mathrm{E}+04$ & $4.40 \mathrm{E}-02$ & $4.54 \mathrm{E}+02$ & 970805 \\
\hline \multicolumn{4}{|c|}{ Sum of the ratios of the releasable quantities $\times 5 \mathrm{E}-03^{\mathrm{a}}$} & $3 \mathrm{E}-02$ \\
\hline
\end{tabular}

Notes:

a. The factor $5 \mathrm{E}-03$ is the ratio of the release fraction of $1.0 \mathrm{E}-03$ inherent in the TQs and RQs to the release fraction of $5.0 \mathrm{E}-06$ (per telecon with $\mathrm{H}$. Goldin regariding Draft EM Hazard Categorization Standard). 
Table A-5. Sum of the ratios for radionuclides found in OU 3-7 soil.

\begin{tabular}{|c|c|c|c|c|c|c|c|c|}
\hline Contaminant & $\begin{array}{c}\text { Assumed soil } \\
\text { concentration } \\
(\mathrm{pCi} / \mathrm{g})\end{array}$ & $\begin{array}{l}\text { Assumed } \\
\text { quantity } \\
\text { (Ci) }\end{array}$ & $\begin{array}{l}\text { ARF used to } \\
\text { derive Cat. } 3 \\
\text { TQs and RQs }{ }^{A-3}\end{array}$ & $\begin{array}{c}\mathrm{TQ} / \mathrm{RQ} \\
\text { adjustment factor } \\
(\mathrm{ARF} / 5 \mathrm{E}-06) \\
\end{array}$ & $\begin{array}{c}\text { Adjusted Cat. } 3 \text { TQs } \\
(\mathrm{TQ} * \text { ARF/5E-06) } \\
(\mathrm{Ci}) \\
\end{array}$ & $\begin{array}{l}\text { Ratio of assumed } \\
\text { quantity to } \\
\text { adjusted TQs } \\
\end{array}$ & $\begin{array}{c}\text { Adjusted RQs } \\
\text { (RQ*ARF/5E-06) } \\
\text { (Ci) } \\
\end{array}$ & $\begin{array}{l}\text { Ratio of assumed } \\
\text { quantity adjusted } \\
\text { RQs } \\
\end{array}$ \\
\hline Americium-241 & $1.50 \mathrm{E}+06$ & $2.25 \mathrm{E}+00$ & $1.00 \mathrm{E}-03$ & $2.00 \mathrm{E}+02$ & $2.00 \mathrm{E}+03$ & $113 E-03$ & $2.00 \mathrm{E}+00$ & $113 \mathrm{E}+00$ \\
\hline Cerium-144 & $2.40 \mathrm{E}+03$ & $3.60 \mathrm{E}-03$ & $1.00 \mathrm{E}-02$ & $2.00 \mathrm{E}+03$ & $2.00 \mathrm{E}+05$ & $1.80 \mathrm{E}-08$ & $2.00 \mathrm{E}+03$ & $180 \mathrm{E}-06$ \\
\hline Cesium-134 & $7.40 \mathrm{E}+04$ & $1.11 \mathrm{E}-01$ & $1.00 \mathrm{E}-02$ & $2.00 \mathrm{E}+03$ & $3.08 \mathrm{E}+06$ & $3,60 \mathrm{E}-08$ & $2.00 \mathrm{E}+03$ & $555 \mathrm{E}-05$ \\
\hline Cesium-137 & $1.00 \mathrm{E}+08$ & $1.50 \mathrm{E}+02$ & $1.00 \mathrm{E}-02$ & $2.00 \mathrm{E}+03$ & $3.16 \mathrm{E}+06$ & $4.75 E-05$ & $2.00 \mathrm{E}+03$ & $750 \mathrm{E}-02$ \\
\hline Cobalt -57 & $1.00 \mathrm{E}+00$ & $1.50 \mathrm{E}-06$ & $1.00 \mathrm{E}-03$ & $2.00 \mathrm{E}+02$ & $1.20 \mathrm{E}+06$ & $125 \mathrm{E}-12$ & $2.00 \mathrm{E}+04$ & $7,50 \mathrm{E}-11$ \\
\hline Cobalt- 60 & $2.30 \mathrm{E}+04$ & $3.45 \mathrm{E}-02$ & $1.00 \mathrm{E}-03$ & $2.00 \mathrm{E}+02$ & $7.20 \mathrm{E}+04$ & $479 \mathrm{E}-07$ & $2.00 \mathrm{E}+03$ & $173 \mathrm{E}-05$ \\
\hline Europium-152 & $8.80 \mathrm{E}+04$ & $1.32 \mathrm{E}-01$ & $1.00 \mathrm{E}-02$ & $2.00 \mathrm{E}+03$ & $4.00 \mathrm{E}+05$ & $330 \mathrm{E}-07$ & $2.00 \mathrm{E}+04$ & $660 \mathrm{E}-06$ \\
\hline Europium-154 & $5.70 \mathrm{E}+05$ & $8.55 \mathrm{E}-01$ & $1.00 \mathrm{E}-02$ & $2.00 \mathrm{E}+03$ & $4.00 \mathrm{E}+05$ & $214 \mathrm{E}-06$ & $2.00 \mathrm{E}+04$ & $428 \mathrm{E}-05$ \\
\hline Europium-155 & $9.60 \mathrm{E}+03$ & $1.44 \mathrm{E}-02$ & $1.00 \mathrm{E}-02$ & $2.00 \mathrm{E}+03$ & $4.00 \mathrm{E}+05$ & $3.60 \mathrm{E}-08$ & $2.00 \mathrm{E}+04$ & $720 \mathrm{E}-07$ \\
\hline Neptunium-237 & $5.50 \mathrm{E}+00$ & $8.25 \mathrm{E}-06$ & $1.00 \mathrm{E}-03$ & $2.00 \mathrm{E}+02$ & $8.40 \mathrm{E}+01$ & $982 \mathrm{E}-08$ & $2.00 \mathrm{E}+00$ & $413 E-06$ \\
\hline Plutonium-241 & $1.10 \mathrm{E}+06$ & $1.65 \mathrm{E}+00$ & $1.00 \mathrm{E}-03$ & $2.00 E+02$ & $6.40 \mathrm{E}+03$ & $258 \mathrm{E}-04$ & $2.00 \mathrm{E}+02$ & $825 \mathrm{E}-03$ \\
\hline Plutonium-242 & $3.20 \mathrm{E}+01$ & $4.80 \mathrm{E}-05$ & $1.00 \mathrm{E}-03$ & $2.00 \mathrm{E}+02$ & $1.24 \mathrm{E}+02$ & $3.87 \mathrm{E}-07$ & $2.00 \mathrm{E}+00$ & $240 \mathrm{E}-05$ \\
\hline Plutonium-238 & $2.70 \mathrm{E}+05$ & $4.05 \mathrm{E}-01$ & $1.00 \mathrm{E}-03$ & $2.00 \mathrm{E}+02$ & $1.24 \mathrm{E}+02$ & $3.27 \mathrm{E}-03$ & $2.00 \mathrm{E}+00$ & $2.03 \mathrm{E}-01$ \\
\hline Plutonium-239/240 & $9.00 \mathrm{E}+04$ & $1.35 \mathrm{E}-01$ & $1.00 \mathrm{E}-03$ & $2.00 \mathrm{E}+02$ & $1.04 \mathrm{E}+02$ & $130 \mathrm{E}-03$ & $2.00 \mathrm{E}+00$ & $675 \mathrm{E}-02$ \\
\hline Ruthenium-106 & $5.40 \mathrm{E}+01$ & $8.10 \mathrm{E}-05$ & $1.00 \mathrm{E}-02$ & $2.00 \mathrm{E}+03$ & $2.08 \mathrm{E}+05$ & $389 \mathrm{E}-10$ & $2.00 \mathrm{E}+03$ & $405 \mathrm{~B}-08$ \\
\hline Strontium-90 & $5.70 \mathrm{E}+07$ & $8.55 E+01$ & $1.00 \mathrm{E}-02$ & $2.00 \mathrm{E}+03$ & $3.20 \mathrm{E}+02$ & $2.67 \mathrm{E}-01$ & $2.00 \mathrm{E}+02$ & $4.28 \mathrm{E}-01$ \\
\hline Tritium & $2.50 \mathrm{E}+04$ & $3.75 \mathrm{E}-02$ & NA & NA & $1.00 \mathrm{E}+03^{\mathrm{b}}$ & $375 \mathrm{E} 05^{\mathrm{b}}$ & $1.00 \mathrm{E}+02^{\mathrm{b}}$ & $375 \mathrm{E}-04^{\mathrm{b}}$ \\
\hline Uranium-234 & $2.10 \mathrm{E}+0 \mathrm{I}$ & $3.15 \mathrm{E}-05$ & $1.00 \mathrm{E}-03$ & $2.00 \mathrm{E}+02$ & $8.40 \mathrm{E}+02$ & $375 \mathrm{E}-08$ & $2.00 \mathrm{E}+01$ & $158 \mathrm{E}-06$ \\
\hline Uranium-235 & $6.40 \mathrm{E}+03$ & $9.60 \mathrm{E}-03$ & $1.00 \mathrm{E}-03$ & $2.00 \mathrm{E}+02$ & $8.40 \mathrm{E}+02$ & $1,14 \mathrm{E}-05$ & $2.00 \mathrm{E}+01$ & $480 \mathrm{E}-04$ \\
\hline Uranium-236 & $7.60 \mathrm{E}-01$ & $1.14 \mathrm{E}-06$ & $1.00 \mathrm{E}-03$ & $2.00 \mathrm{E}+02$ & $8.40 \mathrm{E}+02$ & $136 \mathrm{E}-0$ & $2.00 \mathrm{E}+01$ & $5.70 \mathrm{E}-08$ \\
\hline & & & Sum or the ratic & of assumed concentr & ions to adjusted TQs & $3 \mathrm{E}-01$ & & \\
\hline \multicolumn{8}{|c|}{ Sum of the ratios of assumed concentrations to adjusted RQs } & $2 \mathrm{E}+00$ \\
\hline
\end{tabular}

\section{Notes:}

a The 5.0E-06 is derived from reference A-2 and is appropriate for contaminated soil (per telecon with H. Goldin regariding Draft EM Hazard Categorization Standard).

b. The TQ and RQ for tritium were not adjusted. 


\section{Column C of Table A-1, Table A-3, and Table A-5}

The following calculations were used to determine the assumed quantity of contaminant in $1 \mathrm{~m}^{3}$ of soil containing the assumed concentrations (AC).

$$
\begin{aligned}
& \mathrm{Ci}=\mathrm{AC}\left(\frac{\mathrm{pCi}}{\mathrm{g}}\right) \times \frac{10^{-12} \mathrm{Ci}}{\mathrm{pCi}} \times \frac{1.5 \mathrm{~g}}{\mathrm{~cm}^{3}} \times \frac{10^{6} \mathrm{~cm}^{3}}{\mathrm{~m}^{3}} \times 1 \mathrm{~m}^{3} \\
& \mathrm{~kg}=\mathrm{AC}\left(\frac{\mathrm{mg}}{\mathrm{kg}}\right) \times \frac{10^{-6} \mathrm{~kg}}{\mathrm{mg}} \times \frac{1,500 \mathrm{~kg}}{\mathrm{~m}^{3}} \times 1 \mathrm{~m}^{3}
\end{aligned}
$$

\section{Column C of Table A-2 and Table A-4}

The following calculations were used to determine the assumed quantity of contaminant in 4,000 $L$ of water contaminated with the AC.

$$
\begin{aligned}
& \mathrm{Ci}=\mathrm{AC}\left(\frac{\mathrm{pCi}}{\mathrm{L}}\right) \times \frac{10^{-12} \mathrm{Ci}}{\mathrm{pCi}} \times 4,000 \mathrm{~L} \\
& \mathrm{~kg}=\mathrm{AC}\left(\frac{\mu \mathrm{g}}{\mathrm{L}}\right) \times \frac{10^{-9} \mathrm{~kg}}{\mu \mathrm{g}} \times 4,000 \mathrm{~L}
\end{aligned}
$$




\section{REFERENCES}

A-1. U.S. Department of Energy, Idaho Operations, "Track 2 Sites: Guidance for assessing Low Probability Hazard Sites at the INEL,” DOE/ID-10389, Revision 6, January 1994.

A-2. U.S. Department of Energy, "Airborne Release Fractions/Rates and Respirable Fractions for NonReactor Nuclear Facilities: Volume I - Analysis of Experimental Data,"

DOE-HDBK-3010-94, December 1994.

A-3. ICF Incorporated, Technical Background Document to Support Final Rulemaking Pursuant to Section 102 of the Comprehensive Environmental Response, Compensation, and Liability Act: Radionuclides; A Report to the Emergency Response Division, Office of Emergency and Remedial Response, U. S. Environmental Protection Agency, February 1989. 